\title{
Spending Natural Resource Revenues in an Altruistic Growth Model
}

\author{
Frederiksen, Elisabeth Hermann
}

Publication date:

2006

Document version

Publisher's PDF, also known as Version of record

Citation for published version (APA):

Frederiksen, E. H. (2006). Spending Natural Resource Revenues in an Altruistic Growth Model. Department of Economics, University of Copenhagen. http://www.econ.ku.dk/eprn_epru/Workings_Papers/wp-06-09.pdf 


\section{EPRU Working Paper Series}

Economic Policy Research Unit

Department of Economics

University of Copenhagen

Studiestræde 6

DK-1455 Copenhagen K

DENMARK

Tel: (+45) 35324411

Fax: (+45) 35324444

Web: http://www.econ.ku.dk/epru/

Spending Natural Resource Revenues in an Altruistic Growth Model

Elisabeth Hermann Frederiksen

2006-09

ISSN 0908-7745

The activities of EPRU are financed by a grant from The National Research Foundation 


\title{
Spending Natural Resource Revenues in an Altruistic Growth Model
}

\author{
Elisabeth Hermann Frederiksen* \\ University of Copenhagen, EPRU, ${ }^{\dagger}$ and FAME $^{\ddagger}$
}

26 September 2006

\begin{abstract}
This paper examines how revenues from a natural resource interact with growth and welfare in an overlapping generations model with altruism. The revenues are allocated between public productive services and direct transfers to members of society by spending policies. We analyze how these policies influence the dynamics, and how the dynamics are influenced by the abundance of the revenue. Abundant revenues may harm growth, but growth and welfare can be oppositely affected. We also provide the socially optimal policy. Overall, the analysis suggests that variation in the strength of altruism and in spending policies may be part of the reason why natural resources seem to affect economic performance across nations differently.
\end{abstract}

Keywords: Natural Resources, Economic Growth, Welfare, Altruism

JEL Classification Codes: D64, O41, Q33, Q38

*I wish to thank Carl-Johan Dalgaard, Massimo Franchi, Christian Groth, Christian Schultz, Ragnar Torvik, seminar participants at the SURED 2006, at the EPRU seminar at the University of Copenhagen, and at the 2005 annual DGPE workshop for their helpful discussions and suggestions. Correspondence: Elisabeth Hermann Frederiksen, Department of Economics, University of Copenhagen, Studiestræde 6, 1455 Copenhagen - K, Denmark. E-mail: ehf@econ.ku.dk.

${ }^{\dagger}$ The activities of EPRU (Economic Policy Research Unit) are financed through a grant from The Danish National Research Foundation.

${ }^{\ddagger}$ FAME (Fisheries \& Aquaculture, Management \& Economics) is a network and resource school within economics, and resource and fisheries management at the University of Southern Denmark. 


\section{Introduction}

The idea of a resource curse ${ }^{1}$ is not new; it dates back in history. The decline of Spain's prosperity after its colonization of the New World and discovery of large amounts of gold and other precious metals is a classical example. Also within recent decades, has the idea of a resource curse received support by a large body of empirical (see Auty 1993, 2001; Sachs and Warner 1995, 1999, 2001, among others) as well as theoretical literature. ${ }^{2}$ Classical theories include the Dutch disease theory (Corden and Neary 1982; Torvik 2001; van Wijnbergen 1984), rent seeking problems (Tornell and Lane 1999; Torvik 2002), and political economy explanations (Ross 2004, 2006; Robinson and Torvik 2005). Rodriguez and Sachs (1999) suggest that natural resource rich countries are overshooting their consumption levels and consequently converge to their steady states from above, which results in slow rates of economic growth.

Empirical evidence, however, which questions an unconditional negative relationship between natural resources and growth, seems also to be emerging. Stijns (2005) examines the effect of natural resource abundance on growth rates in a cross-country study by decomposing natural resources into four groups: oil and gas, coal, minerals, and land. He concludes that only land is negatively correlated with economic growth. Sala-i-Martin et al. (2004) investigate overall determinants of growth rates by means of cross-country regressions. Among the robust and significant variables contributing positively to economic growth is the fraction of GDP in

\footnotetext{
${ }^{1}$ We use the term "resource curse" to describe the situation in which resource abundant nations grow slower than nations endowed with fewer resources. In the literature, the term is sometimes used in a more general way to describe poor economic performance. For our analysis, however, it is important to distinguish between growth and welfare effects.

${ }^{2}$ For a recent survey of the literature, consult Stevens (2003).
} 
mining. Besides, a classical counterexample to the resource curse is oil-rich Norway. Larsen (2003) concludes that resources are a blessing for Norway's economy. ${ }^{3}$

Yet only a limited number of theoretical studies have tried to explain a diverging experience in resource impact on economic performance. Exception includes Mehlum et al. (2006). This paper argues that growth performance varies with how resource revenues are distributed between "grabbing" and production, which, in turn, depends on the type of institution. The paper empirically supports that the resource curse is weaker, or completely missing, in countries with producer friendly institutional quality. Another paper by Torvik (2001) proposes a Dutch disease model which explains the variation in resource impact on economic outcomes by differences in learning by doing effects across sectors. In general, however, while there has been intense focus on analyzing natural resources in positive settings, an important aspect, how best to manage the resource revenues despite potential harmful growth effects, has been largely ignored. ${ }^{4}$

Motivated by what could look like an empirical rejection of a pandemic resource curse, this paper takes a closer look at how cross-country variation in the relationship between growth performance and natural resources can be explained, and, in addition, how this relationship relates to welfare.

Revenues from natural resources are typically managed by governments, and political economic factors are likely to influence how revenues are spent. Spending policies, in turn, possibly matters for how revenues impact economic performance.

\footnotetext{
${ }^{3} \mathrm{He}$ notes, however, a slow-down in growth after the mid-90s.

${ }^{4}$ One exception is Matsen and Torvik (2005). They analyze an optimal intertemporal consumption path in a Dutch disease model, and show that the growth maximizing policy differs from the welfare maximizing policy. In their framework, this means some Dutch disease is optimal. Within the literature of exhaustible natural resources, the literature of how optimally to manage resource revenues in order to achieve intergenerational equity is well established, see, e.g., Hartwick (1977) and Solow $(1974,1986)$.
} 
The political economy literature often argues that abundant natural resources lead to poor spending policies. The idea is that "easy" revenues corrupt, bring about conflicts (Ross 2004, 2006), and encourage economically inefficient - but politically important - projects (Robinson and Torvik 2005). To mitigate such problems, Sala-i-Martin and Subramanian (2003) suggest, at least for the case of Nigeria, to decentralize revenues by distributing them directly to the people by which the government is forced to finance public services by taxes. Taxes may be costly to collect, yet overall society gains in that collecting taxes is claimed to incorporate a disciplining mechanism which protects against wasteful projects. ${ }^{5}$

We argue that nations may be in different stages of economic development, or what we refer to as different economic growth regimes, and that across such stages, economic factors as private savings differ in the way they are generated. Insofar that savings matter for growth, decentralized revenues may therefore have different impacts on economic development.

We model the possibility of different growth regimes, and the possibility of different spending policies in a unified framework. We use a two-period overlapping generations growth model in which individuals are altruistic in that parents care about the welfare of their children. Parents have the possibility to leave bequests, which they will do, when their altruism is sufficiently high. In this case, the economy is dynastic and behaves like a representative agent infinitely-lived agent model, whereas the economy behaves like an overlapping generations model, when altruism is not intense enough and bequests are absent (Barro 1974; Weil 1987). Resource

\footnotetext{
${ }^{5} \mathrm{~A}$ similar proposal is made by Sandbu (2006). He argues that tax revenues differ from resource revenues in that the first is considered as out-of-pocket losses and the latter as forgone gains by members of society. In general, he argues, members of society are more likely to hold the government accountable for out-of-pocket losses than for forgone rents.
} 
revenues enter the model in every period as a fixed fraction of man-made output. They are allocated according to a spending policy as direct transfers to members of society and as expenditures on a public productive service as in Barro (1990).

Our results suggest a potential caveat to decentralizing resource revenues. While trying to avoid a resource curse created through political economy mechanisms by distributing revenues directly to members of society, a resource curse may be created due to economic factors instead. In addition, we examine various endogenous spending policies and find that under such policies, increased resource abundance may lead to a shift in growth regime to a regime with a lower growth rate, as such a shift implies higher welfare

Our model is related to that of Papyrakis and Gerlagh (2004), but more general. Papyrakis and Gerlagh (2004) study a two-generation overlapping generations model (without altruism), in which resource revenues are given entirely to the retired old generation. Higher revenues means less savings, and therefore the economy is resource cursed. ${ }^{6}$ Our study emphasizes that the resource curse is fragile with respect to variation in the allocation of revenue across generations, and that potential adverse effects on savings can be remedied by spending policies that stimulate intergenerational transfers.

Our model is also related to the literature that studies effectiveness of economic policy in an altruistic setting. Caballe (1998) analyzes how taxation of labor and capital influences not only growth performance, but also the growth regime. In his model, the level of altruism that distinguishes the growth regimes is determined by

\footnotetext{
${ }^{6}$ The authors consider in an appendix a situation, in which all individuals (i.e., young and old) equally divide the resource revenue and find that resource revenues are less harmful to savings than when revenues are only given to the old.
} 
the tax policy. Croix and Michel (2002, ch. 5) analyze the neutrality of economic policy, when the bequest motive is operative.

The paper proceeds as follows. We present the model in section 2 . In section 3 , we explain the market equilibrium and characterize the conditions for the altruism factor that distinguishes the growth regimes. In section 4, we examine different policy objectives, derive corresponding spending policies, and analyze the impact of natural resource revenues on growth and welfare under these policies. In section 5, we study the optimal policy, and the final section provides concluding remarks.

\section{The Model}

The economy is closed and described by a one sided altruistic overlapping generations' framework. Parents care about the welfare of their offspring and have the possibility to make intergenerational transfers to their immediate descendants in the form of bequests. Individuals live for two periods: as young and as old. Only the young generation works, the old generation is retired. There are $L$ individuals in each generation, which remains constant over time.

\subsection{Natural Resource Revenues}

In every period $t$, revenues from the sale of a natural resource enter the economy. The value of the revenue is exogenously given as a fixed fraction, $\xi$, of the real value of man-made output, $Y_{t}$, where $0<\xi<1$. We may think of $\xi$, which we refer to as resource abundance, as a characteristic that is country specific. ${ }^{7}$

\footnotetext{
${ }^{7}$ Natural resource revenues vary considerably across countries. For instance, Iceland, Nigeria, Norway and Venezuela have a share of primary exports in GDP above 0.2, whereas Nepal, Sweden and the US have a share of primary exports below 0.1. (http://www.cid.havard.edu/ciddata/ciddata.html.)
} 
Let $E_{t}$ denote the real value of the revenue; then,

$$
\xi=\frac{E_{t}}{Y_{t}}
$$

Accordingly, our theoretical model applies also to inflows of foreign aid and other gifts and transfers from "abroad." As the resource revenue man-made output ratio is constant over time, we focus purely on spending policies in relation to intergenerational transfers and economic growth. ${ }^{8}$ Similar ways of modeling of the revenue (or foreign aid) are found in Chatterjee et al. (2003), Lensink and White (2001), Papyrakis and Gerlagh (2004), and Torvik (2001). ${ }^{9}$

\subsection{Spending Policies}

Based on a spending policy, a government spends all resource revenues in every period on one or two purposes.

First, it may allocate a share, $\tau$, where $0 \leq \tau<1$, directly to members of society in a lump-sum fashion. ${ }^{10}$ Of this share, the young share parameter, $\pi$, is given to the young generation and $(\tau-\pi)$ to the old; i.e., $0 \leq \pi \leq \tau$.

Second, the government invests the remaining resource revenue in a public service flow, $G_{t}$, that works as input into production. We think of the public service as a broad range of services that could be infrastructure, administration, legal, environmental services, etc. There are no externalities associated with the use of public services

\footnotetext{
${ }^{8}$ For a reference on optimal resource extraction, consult Dasgupta and Heal (1979).

${ }^{9}$ Torvik (2001) discusses alternative ways of modeling the revenue in footnote 4, p. 290 . The important assumption is that the revenue grows over time so that, as a share of income, it does not converge towards zero.

${ }^{10} \mathrm{~A}$ real example of direct transfers of resource rents is found in Alaska. One purpose of the socalled Alaska Permanent Fund is to distribute the returns of the fund, which come from minerals and oil, to all inhabitants of the state in the form of a check (Hannesson 2001).
} 
In every period, the government runs a balanced budget. It cannot issue debts nor run surpluses by accumulating assets. Hence,

$$
G_{t}=(1-\tau) E_{t}=(1-\tau) \xi Y_{t}
$$

Therefore, the resource constraint, which is the public budget, satisfies

$$
\pi E_{t}+(\tau-\pi) E_{t}+(1-\tau) E_{t}=E_{t}=\xi Y_{t}
$$

\section{$2.3 \quad$ Firms}

A representative firm produces man-made output, $Y_{t}$, and uses three factors in production: labor, $L$, the average public service flow per worker, $g_{t} \equiv G_{t} / L$, and capital, $K_{t}$. Output per worker, $y_{t}$, is produced according to the following production technology:

$$
y_{t}=A g_{t}^{\alpha} k_{t}^{1-\alpha}
$$

where $0<\alpha<1$ is the share of labor and of public services in production, $A$ is a positive constant productivity term, and $k_{t}$ is capital per worker. Labor productivity increases as the public service flow per worker, $g_{t}$, increases. ${ }^{11}$

The representative firm maximizes profits taking $g_{t}$, as well as the price of output, which is the numeraire, and of inputs, as given. Capital fully depreciates in each period, and each factor is paid its private marginal product.

$$
\begin{aligned}
\frac{\partial Y_{t}}{\partial K_{t}} & =(1-\alpha) A\left(\frac{g_{t} L}{K_{t}}\right)^{\alpha}=1+r_{t} \\
\frac{\partial Y_{t}}{\partial L_{t}} & =\alpha A\left(\frac{g_{t} L}{K_{t}}\right)^{\alpha} k_{t}=w_{t}
\end{aligned}
$$

where $r_{t}$ is the rental rate of capital, and $w_{t}$ is the wage rate.

\footnotetext{
${ }^{11}$ The public service flow per worker is non-rival, but subject to congestion from $L$.
} 


\subsection{Altruistic Individuals}

Newborn individuals are identical within as well as across generations. A parent is altruistic with respect to the welfare of her offspring in the Barro (1974) sense and weights her offspring's utility in her utility function, $V_{t}$. Let $U_{t}$ denote utility derived from life-cycle consumption; thus, total utility of an individual at time $t$ can be presented as

$$
V_{t}=U_{t}+\beta V_{t+1}
$$

where $0<\beta<1$ is the intergenerational discount factor, which we refer to as the altruism factor. When generations are altruistic, parents care about the welfare of their children, who in turn care about the welfare of their children, and so forth. In this way, welfare of all future generations is linked.

Utility from own consumption is the sum of utility from consumption as young, $c_{1 t}$, and the discounted utility of consumption as old, $c_{2 t+1}$. Specifically,

$$
U_{t}=u\left(c_{1 t}\right)+\rho u\left(c_{2 t+1}\right)=\ln \left(c_{1 t}\right)+\rho \ln \left(c_{2 t+1}\right),
$$

where $0<\rho<1$ is the intertemporal discount factor. By recursively eliminating $V_{t+i}, i=0, \ldots, \infty$, in $(7)$ we have. ${ }^{12}$

$$
V_{t}=\sum_{i=0}^{\infty} \beta^{i}\left[\ln \left(c_{1 t+i}\right)+\rho \ln \left(c_{2 t+1+i}\right)\right]
$$

saying that utility of a young individual born at time $t$ equals own life-cycle utility plus the discounted sum of life-cycle utilities of her descendants.

\footnotetext{
${ }^{12}$ Eq. (7) can be rewritten by induction as $V_{t}=\sum_{i=0}^{T} \beta^{i}\left[u\left(c_{1 t+i}\right)+\rho u\left(c_{2 t+1+i}\right)\right]+\beta^{T+1} V_{t+1+T}$ Taking the limit for $T \rightarrow \infty$ and assuming that total utility satisfy the limit condition $\lim _{T \rightarrow \infty} \beta^{T+1} V_{t+1+T}=0$, we get $V_{t}=\sum_{i=0}^{T} \beta^{i}\left(U_{t+i}\right)$. Using $U_{t}=\ln \left(c_{1 t}\right)+\rho \ln \left(c_{2 t+1}\right)$, we have (9).
} 
In any period $t$, the young individual inelastically supplies one unit of labor for which she receives the market wage rate, $w_{t}$. When the young share parameter, $\pi$, is positive, she also receives a direct transfer as a share of the natural resource revenue, and, finally, she may inherit bequests, $b_{t}$, from her parents. She consumes $c_{1 t}$ and saves $s_{t}$ for her retirement; hence,

$$
c_{1 t}+s_{t}=b_{t}+w_{t}+\pi e_{t},
$$

where $\pi e_{t} \equiv \pi E_{t} / L$ denotes the lump-sum resource revenue income of a young at time $t$. When old, she receives the proceeds of her saving, $\left(1+r_{t+1}\right) s_{t}$, where $r_{t+1}$ is the rate of interest. In addition, if $\pi<\tau$, she receives income from the natural resource, which she consumes and possibly bequeaths to her offspring. Accordingly, her period two budget constraint can be written as

$$
c_{2 t+1}+b_{t+1}=\left(1+r_{t+1}\right) s_{t}+(\tau-\pi) e_{t+1},
$$

where $(\tau-\pi) e_{t+1}$ is the resource revenue given lump-sum to an old person at time $t+1$. Bequests cannot be negative, i.e., $b_{t+1} \geq 0$. This restriction prevents parents from leaving debts to their children.

The dynamics of bequests are found by eliminating $s_{t}$ in (10) and (11):

$$
b_{t+1}=\left(1+r_{t+1}\right)\left(b_{t}+w_{t}+\pi e_{t}-c_{1 t}\right)+(\tau-\pi) e_{t+1}-c_{2 t+1} .
$$

An individual of generation $t$ maximizes life time utility given in (9) subject to the two budget constraints, (10) and (11), and the non-negativity constraint on bequests, by optimally choosing consumption, savings, and bequests, taking $b_{t}, w_{t}$, $r_{t+1}, e_{t}$, and $e_{t+1}$ as given.

The Lagrangian of period $t$ is equal to life-cycle utility, $U_{t}$, with the change $\beta p_{t+1} b_{t+1}-p_{t} b_{t}$ in the shadow value of bequests, $p_{t}$, over a period (Croix and Michel 
$2002,244)$

$$
\mathcal{L}_{t}=\ln \left(c_{1 t}\right)+\rho \ln \left(c_{2 t+1}\right)+\beta p_{t+1} b_{t+1}-p_{t} b_{t}
$$

Note that $b_{t+1} \geq 0$ implies $\left(1+r_{t+1}\right)\left(b_{t}+w_{t}+\pi e_{t}\right)+(\tau-\pi) e_{t+1} \geq\left(1+r_{t+1}\right) c_{1 t}+$ $c_{2 t+1}$. Incorporating this restriction in the maximization problem, the optimality conditions, which are both necessary and sufficient, are given by

$$
\frac{1}{c_{1 t}}=\frac{\rho\left(1+r_{t+1}\right)}{c_{2 t+1}}
$$

and

$$
b_{t+1}\left(\frac{\beta}{c_{1 t+1}}-\frac{\rho}{c_{2 t+1}}\right) \leq 0\left(=0 \text { if } b_{t+1}>0\right) .
$$

The transversality condition is

$$
\lim _{t \rightarrow \infty} \beta^{t} p_{t} b_{t}=0
$$

Equation (14) describes the trade-off between a person's consumption as young and as old. In optimum, the individual is indifferent between consuming as young and saving for old consumption. Equation (15) says that when bequests are positive, a parent's marginal utility of own consumption equals her marginal utility of the offspring's consumption. If a parent's marginal utility from her offspring's consumption is less than the marginal utility of her own consumption, then bequests are zero, and the solution is given by a corner solution.

\section{Competitive Equilibrium}

For simplicity, we normalize the number of working people, $L$, to unity, so we can write $E_{t}=e_{t}, Y_{t}=y_{t}, K_{t}=k_{t}$, and $G_{t}=g_{t}$. We obtain the following expression by rewriting (4) using (2):

$$
y_{t}=A k_{t}\left[\frac{(1-\tau) \xi y_{t}}{k_{t}}\right]^{\alpha} \Leftrightarrow y_{t}=\left[A(1-\tau)^{\alpha} \xi^{\alpha}\right]^{\frac{1}{1-\alpha}} k_{t} \equiv f(\tau, \xi) k_{t}
$$


where $\frac{\partial f(\tau, \xi)}{\partial \tau}<0$. The larger the share of the natural resource revenues spent on direct transfers, the smaller the public service flow. This implies a smaller public service flow capital ratio, $\frac{g_{t}}{k_{t}}$. Due to the AK structure of the model, it also leads to a drop in the output capital ratio. Therefore, all things equal, $\frac{\partial f(\tau, \xi)}{\partial \xi}>0$; higher revenues increase public services.

Using (4), factor market clearing implies

$$
\begin{aligned}
r_{t} & =(1-\alpha) A\left(\frac{g_{t}}{k_{t}}\right)^{\alpha}-1, \\
w_{t} & =\alpha A\left(\frac{g_{t}}{k_{t}}\right)^{\alpha} k_{t}
\end{aligned}
$$

and using (17) in (18) and (19), we get

$$
\begin{aligned}
r_{t} & =(1-\alpha) f(\tau, \xi, \cdot)-1 \equiv r(\tau, \xi) \\
w_{t} & =\alpha f(\tau, \xi, \cdot) k_{t} \equiv w(\tau, \xi) k_{t}
\end{aligned}
$$

where $w(\tau, \xi)$ denotes the wage rate capital ratio. Both the rate of return and the wage rate are positively associated with the public service flow capital ratio, $\frac{g_{t}}{k_{t}}$, and, thus, $\frac{\partial r(\tau, \xi)}{\partial \tau}<0$ and $\frac{\partial w(\tau, \xi)}{\partial \tau}<0$.

The capital market equilibrium requires savings of the young to equal capital installed in the productive sector:

$$
s_{t}=k_{t+1}
$$

Lastly, the goods market equilibrium is given by the aggregate resource constraint. Using the budget constraints (10), and (11), and the equilibrium conditions (2), (20), (21), and (22), the aggregate resource constraint can be expressed as

$$
(1+\xi) y_{t}=c_{1 t}+c_{2 t}+k_{t+1}+g_{t}
$$


Total income in period $t$ is the sum of man-made output plus the natural resource revenue.

\subsection{Dynamics}

In the following, we distinguish two growth regimes of the economy based on the presence of intergenerational transfers. When parents marginal utility of own consumption is larger than the marginal utility they derive from the offspring's consumption, the non-negativity constraint on bequests is binding, and there are no bequests.

\subsubsection{Zero Bequests}

Assume that (15) holds with inequality so bequests are absent. Letting $b_{t}=b_{t+1}=0$ in (10) and (11), we can, by also using (14), derive the an expression for the savings $s_{t}:$

$$
s_{t}=\frac{\rho}{1+\rho}\left[w(\tau, \xi) k_{t}+\pi e_{t}\right]-\frac{(\tau-\pi) e_{t+1}}{(1+\rho)[1+r(\tau, \xi)]} .
$$

Savings are increasing in wages and resource revenues received as young and decreasing in resource revenues received as old. This is intuitive; consumption smoothing requires higher savings the smaller income is as old compared to income as young.

Using (22), we get from (24)

$$
k_{t+1}=\frac{\rho}{1+\rho}\left[w(\tau, \xi) k_{t}+\pi e_{t}\right]-\frac{(\tau-\pi) e_{t+1}}{(1+\rho)[1+r(\tau, \xi)]},
$$

which is the law of motion of capital. Dividing both sides by $k_{t}$, we find

$$
\gamma_{t+1}^{O}=\frac{\rho}{1+\rho}\left[w(\tau, \xi)+\pi \frac{e_{t}}{k_{t}}\right]-\frac{(\tau-\pi) \frac{e_{t}}{k_{t}}\left(\gamma_{t+1}^{O}+1\right)}{(1+\rho)[1+r(\tau, \xi)]}-1,
$$

where $\gamma_{t+1}^{O}=\left(\mathrm{k}_{t+1} / k_{t}\right)-1$ is the growth rate of capital (and also capital per worker due to a constant labor force) when bequests are absent. Note that $e_{t}=\xi y_{t}=$ 
$\xi f(\tau, \xi, \cdot) k_{t}$. Rearrange, and $\gamma_{t+1}^{O}$ can be expressed as

$$
\gamma_{t+1}^{O}=\frac{\rho[1+r(\tau, \xi)][w(\tau, \xi)+\pi \xi f(\tau, \xi)]}{(1+\rho)[1+r(\tau, \xi)]+(\tau-\pi) \xi f(\tau, \xi)}-1 \equiv \gamma^{O}(\pi, \tau, \xi)
$$

We define a balanced growth path as a path along which $c_{1 t}, c_{2 t}, k_{t}, y_{t}, g_{t}$, and $e_{t}$ grow at constant relative rates in all periods $t>0$. From (17) it follows that capital grows at the same rate as output. Since resource revenues are given as a fixed fraction of output (in (1)), it follows immediately that also revenues grow at the same rate as output. Moreover, as public services are given as a fixed fraction of total resource revenues (in (2)), public services grow at the same rate as output. From the goods market equilibrium, (23), it follows that aggregate consumption $\left(c_{1 t}\right.$ plus $c_{2 t}$ ) grows at the same rate of output. By $(14),(20)$, and $r_{t}=r(\tau, \xi)$, it then follows that period one and period two consumption grow at the rate of output. Hence, the bequest constrained economy has no transitional economics; $c_{1 t}, c_{2 t}, k_{t}$, $y_{t}, g_{t}$, and $e_{t}$ grow at the same rate along a balanced growth path at all periods $t$.

We denote values taken by the variables on the balanced growth path without bequests with the superscript "O." Using (20), (21), and taking $k_{0}^{O}>0$ as given, equilibrium is given by

$$
\begin{aligned}
c_{1 t}^{O} & =f(\tau, \xi)(\alpha+\pi \xi) \frac{1-\alpha+(\tau-\pi) \xi}{(1+\rho)(1-\alpha)+(\tau-\pi) \xi} k_{t}^{O} \equiv c_{1 t}^{O}(\pi, \tau, \xi), \\
c_{2 t}^{O} & =f(\tau, \xi)[1-\alpha+(\tau-\pi) \xi] k_{t}^{O} \equiv c_{2 t}^{O}(\pi, \tau, \xi), \\
k_{t+1}^{O} & =\frac{\rho(1-\alpha) f(\tau, \xi)(\alpha+\pi \xi)}{(1+\rho)(1-\alpha)+(\tau-\pi) \xi} k_{t}^{O} \equiv k_{t+1}^{O}(\pi, \tau, \xi) .
\end{aligned}
$$

On this growth path, parents behave as if they are selfish as they do not leave intergenerational transfers. Essentially, the economy behaves like an overlapping generations model. 
Growth is positive when income received in period one is sufficiently large to ensure that savings exceed the capital depreciation. Accordingly, $\rho(1-\alpha) f(\tau, \xi)(\alpha+$ $\pi \xi)>(1+\rho)(1-\alpha)+(\tau-\pi) \xi$ implies that $\gamma^{O}(\pi, \tau, \xi)>0$.

\subsubsection{Positive Bequests}

When bequests are positive, (15) holds with equality, and the economy is dynastic. In this regime, the growth rate of period one consumption, $\gamma_{t+1}^{D}$, is found by dividing the first order solutions given in (14) and (15):

$$
\gamma_{t+1}^{D}=\beta[1+r(\tau, \xi)]-1 \equiv \gamma^{D}(\beta, \tau, \xi)
$$

Again, we define a balanced growth path as a path along which $c_{1 t}, c_{2 t}, k_{t}, y_{t}, g_{t}$, $b_{t}$, and $e_{t}$ grow at a constant relative rates in all periods $t>0$. From (17) it follows that capital grows at the same rate as output. Since resource revenues are given as a fixed fraction of output (in (1)), it follows immediately that natural resource revenues grows at the same rate as output. Moreover, as public services are given as a fixed fraction of resource revenues (in (2)), also public services grow at the same rate as output.

By the goods market equilibrium, (23), and $r_{t}=r(\tau, \xi)$, it must be that if capital and output grow as the same rate, then this rate equals that of growth of consumption. From (14), we know that consumption as old and as young is a constant ratio, so old consumption grows at the same rate as young consumption. From either of the budget constraints (10) or (11), it follows that also bequests grow at the same rate as consumption. Thus, also the bequest constrained economy has no transitional economics; $c_{1 t}, c_{2 t}, k_{t}, y_{t}, g_{t}, b_{t}$, and $e_{t}$ grow at the same rate along a balanced growth path at all periods $t$. 
From the first order conditions to (13), it can be shown that $p_{t}$ equals $\frac{1}{c_{1 t}}$, when bequests are positive. ${ }^{13}$ Hence, $p_{t}$ decreases at the rate $\gamma^{D}(\tau, \xi)$. We can thus conclude, when $b_{t}>0, p_{t} b_{t}$ is a constant, and the transversality condition in (16) simplifies to $\beta<1$. When (15) holds with equality, parents leave bequests and the economy behaves like a dynasty of infinitely-lived generations.

We denote values taken by the variables on the balanced growth path with positive bequests with the superscript " $D$." Equilibrium is given by

$$
\begin{aligned}
b_{t}^{D} & =f(\tau, \xi)\left\{\frac{\beta \tau \xi-\rho+[\rho+\beta(1+\rho)](1-\alpha)}{\beta+\rho}-\pi \xi\right\} k_{t}^{D} \equiv b_{t}^{D}(\beta, \tau, \xi),(30) \\
c_{1 t}^{D} & =\beta f(\tau, \xi)\left[\frac{1+\tau \xi-\beta(1-\alpha)}{\beta+\rho}\right] k_{t}^{D} \equiv c_{1 t}^{D}(\beta, \tau, \xi), \\
c_{2 t}^{D} & =\rho f(\tau, \xi)\left[\frac{1+\tau \xi-\beta(1-\alpha)}{\beta+\rho}\right] k_{t}^{D} \equiv c_{2 t}^{D}(\beta, \tau, \xi), \\
k_{t+1}^{D} & =\beta f(\tau, \xi)(1-\alpha) k_{t}^{D} \equiv k_{t+1}^{D}(\beta, \tau, \xi),
\end{aligned}
$$

with $k_{0}^{D}>0$.

Along this growth path, the growth rate is positive when $\beta>\frac{1}{1+r(\tau, \xi)}$. This condition says, for a young individual to have positive savings and bequests, marginal utility of consuming one unit extra as young is less than marginal utility derived from letting the offspring consume $1+r(\tau, \xi)$ units.

Clearly, the growth paths described by (25) and (29) in general differ, as does the way they respond to changes in revenues and in spending policies.

\subsection{The Resource Curse}

The resource curse typically indicates a negative relationship between natural resource abundance and economic performance. In this paper, the resource curse

\footnotetext{
${ }^{13} \mathrm{By}(13), \frac{\partial \mathcal{L}_{t}}{\partial c_{1 t}}=0$ implies that $\frac{1}{c_{1 t}}=\beta p_{t+1}[1+r(\tau, \xi)]$, and $\frac{\partial \mathcal{L}_{t}}{\partial b_{t}}=0$ implies that $p_{t}=$ $\beta p_{t+1}[1+r(\tau, \xi)]$, so it follows that $\frac{1}{c_{1 t}}=p_{t}$.
} 
specifically describes the situation where resource abundant nations grow slower than nations endowed with fewer resources. We can think of two possibilities of why an economy may be "resource cursed": when spending policies are such that increased abundance (i) leads to savings decline, and (ii) leads to a regime shift to a regime with a lower growth rate. In the following, we explain and examine both possibilities.

\subsubsection{Savings Decline}

Indeed, we find that savings may be negatively influenced by increased resource revenues. In particular,

Lemma 1. There exists exogenous policies $(\tau, \pi)$ that imply a resource curse only if at the same time bequests are absent (whether this is due to the policy or a low altruism factor).

Proof. When bequests are absent, along $\gamma^{O}(\xi, \cdot)$ :

$$
\frac{\partial \gamma^{O}(\xi, \cdot)}{\partial \xi}=\frac{\left\{\frac{\partial f(\tau, \xi)}{\partial \xi} \rho(1-\alpha)(\alpha+\pi \xi)+f(\tau, \xi)\left[\rho(1-\alpha) \pi-\frac{\rho(1-\alpha)(\alpha+\pi \xi)(\tau-\pi)}{(1+\rho)(1-\alpha)+(\tau-\pi) \xi}\right]\right\}}{(1+\rho)(1-\alpha)+(\tau-\pi) \xi} .
$$

A policy where $\pi=0$ and $\tau(1-2 \alpha)>\frac{(1-\alpha) \alpha}{\xi}(1+\rho)$ implies $\frac{\partial \gamma^{O}(\xi, \cdot)}{\partial \xi}<0$. In a dynastic growth regime,

$$
\frac{\partial \gamma^{D}(\xi, \cdot)}{\partial \xi}=\beta(1-\alpha) \frac{\partial f(\tau, \xi)}{\partial \xi}>0 \forall 0 \leq \tau<1
$$

proves the non-existence of a resource curse, when bequests are positive.

An operative bequest motive eliminates the resource curse threat as the growth rate in this regime is increasing in the rate of return to capital, which, in turn, is increasing in resource abundance; i.e., $\frac{\partial r(\tau, \xi)}{\partial \xi}>0$. Savings are unaffected by the 
allocation of direct transfers as any change in revenues given to a young individual is offset by an identical opposite change in bequests. ${ }^{14}$ Hence, the rate of growth in this environment, $\gamma^{D}(\beta, \tau, \xi)$, is independent of $\pi$.

When bequests are absent, accumulation of capital depends on the distribution of resource revenues across generations. For example, a policy that distributes all direct transfers solely to the old generation may lead to a resource curse outcome, in which, higher resource abundance results in fewer savings. The resource curse prevails when, due to increased resource abundance, a young individual derives higher marginal utility of consuming as young than as old; i.e., what generates the resource curse is a "disproportional" large direct transfer to the old generation in the situation where bequests are absent. Therefore,

Proposition 1. Given an exogenous policy $(\tau, \pi)$, then when direct transfers from the government are absent, or when they, if present, are allocated only to the young generation, there is no resource curse.

Proof. By lemma 1, we only analyze an economy without bequests. If $\pi=\tau=0$, then

$$
\frac{\partial \gamma^{O}(\xi, \cdot)}{\partial \xi}=\frac{\rho \alpha}{1+\rho} \frac{\partial f(\tau, \xi)}{\partial \xi}>0 \forall \tau=0
$$

and, if $\pi=\tau$, then

$$
\frac{\partial \gamma^{O}(\xi, \cdot)}{\partial \xi}=\frac{\rho}{1+\rho}\left[\frac{\partial f(\tau, \xi)}{\partial \xi}(\alpha+\tau \xi)+f(\tau, \xi) \tau\right]>0 \forall 0<\tau<1
$$

\footnotetext{
${ }^{14}$ To see this notice that along a balanced growth path, eq. (12) can be rewritten as $b_{t}^{D}(\cdot)=\frac{(1+r(\cdot))\left(w(\cdot) k_{t}^{D}+\pi e_{t}^{D}-c_{1 t}^{D}\right)+(\tau-\pi)\left(1+\gamma^{D}(\cdot)\right) e_{t}^{D}-\frac{\rho}{\beta}\left(1+\gamma^{D}(\cdot)\right) c_{1 t}^{D}}{\left(1+\gamma^{D}(\cdot)\right)-(1+r(\cdot))}$, where $\frac{\partial b_{t}^{D}(\cdot)}{\partial \pi}=-e_{t}^{D}$. Now, write $s_{t}=\frac{\rho}{1+\rho}\left(b_{t}^{D}+w(\cdot) k_{t}^{D}+\pi e_{t}^{D}\right)-\frac{(\tau-\pi)\left(1+\gamma^{D}(\cdot)\right) e_{t}^{D}-\left(1+\gamma^{D}(\cdot)\right) b_{t}^{D}}{(1+\rho)(1+r(\cdot))} \equiv s_{t}^{D}(\pi)$. Calculating $\frac{\partial s_{t}^{D}(\pi)}{\partial \pi}$ using $\frac{\partial b_{t}^{D}(\cdot)}{\partial \pi}=-e_{t}^{D}$ gives $\frac{\partial s_{t}^{D}(\pi)}{\partial \pi}=0$.
} 
A larger inflow of revenues, ceteris paribus, enhances the public service flow capital ratio and, thus, the wage rate and the rate of return. When direct transfers are positive, young income increases further relative to old income and savings grow.

We make an interesting observation about the resource curse:

Proposition 2. Increased resource abundance may improve welfare of the current generations, despite causing a resource curse.

Proof. See appendix.

Next, we turn to examine how it can be determined that a particular economy belongs to either of the two growth regimes and how the economy may shift between growth regimes. In particular, we focus on relating these issues to spending policies and to the size of the revenue to man-made output ratio, $\xi$.

\subsubsection{Growth Regime Shifts}

Though the altruism factor, $\beta$, is exogenously given, whether bequests are positive or zero, is determined endogenously by the first order condition given in (15). From (15), we know that when parents marginal utility of own consumption is greater than their marginal utility from the offspring's consumption, the economy is without bequests. A decline in the consumption of the offspring relative to consumption of the parent triggers intergenerational transfers, if the decline is large enough.

We define the threshold value of the altruism factor, $\beta^{*}$, such that under a given spending policy, $(\tau, \pi)$, when $\beta=\beta^{*}$ then $\gamma^{O}(\pi, \tau, \xi)=\gamma^{D}(\beta, \tau, \xi)$. In the special case, where $\beta=\beta^{*}, b_{t}^{D}(\beta, \tau, \xi)=0$ and parents leave zero bequests. Inverting (29), substituting in (25), using (20), and (21) yields

$$
\beta^{*}=\frac{\rho(\alpha+\pi \xi)}{(1+\rho)(1-\alpha)+(\tau-\pi) \xi} \equiv \beta^{*}(\pi, \tau, \xi)
$$


Using this definition of $\beta^{*}$, we obtain the standard result (Caballe 1998; Cardia and Michel 2004; Weil 1987) that, when the altruism factor is less than the threshold value, $\beta<\beta^{*}(\pi, \tau, \xi)$, the economy is without bequest, and the growth path is described by (25). On the other hand, when the altruism factor is higher than the threshold value, $\beta>\beta^{*}(\pi, \tau, \xi)$, bequests are positive, and growth evolves according to (29). We can now compare growth rates in the two regimes:

Proposition 3. Given $\beta \neq \beta^{*}$, then, if the altruism factor ensures positive bequests, the economy grows faster than if the altruism factor leads to absence of bequests.

Proof. We note, by eq. (34), that eq. (25) can be rewritten as $\gamma^{O}(\pi, \tau, \xi)$ $=\beta^{*}(\pi, \tau, \xi)(1-a) f(\tau, \xi)-1$. Since the economy follows this growth path as long as $\beta<\beta^{*}(\pi, \tau, \xi)$, but changes to $\gamma^{D}(\beta, \tau, \xi)=\beta(1-a) f(\tau, \xi)-1$ with positive bequests when $\beta>\beta^{*}(\pi, \tau, \xi)$, it must be that $\gamma^{O}(\pi, \tau, \xi, \cdot)<\gamma^{D}(\beta, \tau, \xi)$.

We observe in particular that $\beta^{*}(\pi, \tau, \xi)$ is a function of the spending policy as well as the size of the natural resource abundance. The larger direct transfers given to the young, $\pi \xi$, the more altruistic the parents must be to leave bequests, and the larger direct transfers given to the old, $(\tau-\pi) \xi$, less altruistic parents also leave bequests. In general, changes in $\xi$ amplify differences in direct transfers across generations, and we give the following direct transfer allocation rules: When $\tau=\pi\left[(1+\rho)\left(\frac{1-\alpha}{\alpha}\right)+1\right]$, there is no effect on $\beta^{*}(\pi, \tau, \xi)$ from changes in $\xi$; i.e., $\frac{\partial \beta^{*}(\pi, \tau, \xi)}{\partial \xi}=0$, and when $\tau<(>) \pi\left[(1+\rho)\left(\frac{1-\alpha}{\alpha}\right)+1\right], \frac{\partial \beta^{*}(\pi, \tau, \xi)}{\partial \xi}>(<) 0$. Therefore, changes in $\xi$ may push economies from one growth regime to another. In the following, we examine what happens to the growth rate when increases in $\xi$ cause 
the economy to shift regime:

Proposition 4. Given the exogenous spending policy $(\tau, \pi)$, when an economy shifts from one growth regime to another growth regime due to increased natural resource abundance, the growth rate goes up.

Proof. See appendix.

Summing up, this section illustrates spending policies matter and that they matter differently depending on the presence of bequests. When spending policies are exogenous, the resource curse prevails as a consequence of savings decline, whereas regime shifts caused by endogenous changes in the threshold altruism factor due to higher resource abundance lead to higher growth rates. Finally, we note that the resource curse and welfare gains may be opposite sides of the same coin.

\section{Political Equilibrium}

It seems reasonable, however, to think of spending policies as typically endogenously determined by a specific economic or political agenda. Therefore, in this section we ask a slightly different question, namely if there are economies in which the resource curse exist under endogenous policies. We examine specific policy objectives.

\subsection{Growth Maximizing Policies}

LeMma 2. Let $\widehat{\pi}^{O}$ and $\widehat{\tau}^{O}$ be the growth maximizing policy when the public budget is given by the resource revenues (in (3)) and the economy is constrained to be without bequests. Then $\widehat{\pi}^{O}=\tau$ and

$$
\widehat{\tau}^{O}=\left\{\begin{array}{lll}
0 & \text { if } & 1-\alpha-\frac{\alpha^{2}}{\xi} \leq 0 \\
1-\alpha-\frac{\alpha^{2}}{\xi} & \text { if } & 1-\alpha-\frac{\alpha^{2}}{\xi}>0
\end{array} .\right.
$$




\section{Proof. See Appendix.}

The intuition for this result is as follows. When direct transfers are positive and given to the young, they influence the growth rate through two channels between which there is a trade-off. The higher $\tau$, the larger direct transfers (to the young generation) which, ceteris paribus, leads to larger savings for retirement. The higher $\tau$, however, the less public service input into private production. Lower public service input into production leads to lower marginal factor productivity, and lower wage rates means fewer savings for retirement. ${ }^{15}$

When $\pi=\widehat{\pi}^{O}=\tau$ and $\tau=\widehat{\tau}^{O} \equiv \widehat{\tau}^{O}(\alpha, \xi)$, savings are maximized under the given public budget and generate the highest feasible growth rate in a no bequest environment. We notice, when the value of $\widehat{\tau}^{O}(\alpha, \xi)$ is given by a corner solution, the rate of economic growth will increase further if the government is able to collect lump-sum taxes to expand the public service. In such a situation the size of the public service flow is sub-optimal.

Notice also $\frac{\partial \widehat{\tau}^{O}(\alpha, \xi)}{\partial \xi}>0$ when $1-\alpha-\frac{\alpha^{2}}{\xi}>0$. This means that the more abundant the resource, a larger share of the revenues is given as direct transfers in order to maximize growth. The reason is that the larger $\xi$, the higher the value of one unit of direct transfer. Higher costs, in terms of lower factor payments, can therefore be tolerated; i.e., the benefits exceed the costs up until the new policy rule.

Lemma 3. Let $\widehat{\tau}^{D}$ be the growth maximizing policy when the public budget is given by the resource revenues (in (3)) and the economy is constrained to be with

\footnotetext{
${ }^{15}$ Moreover, less public service input into production means less man-made output. Since resource revenues are a fixed fraction of total output, this effect feeds back into lowering the total amount of resource revenues to be distributed in the first place. This externality, however, is not internalized in the competitive equilibrium.
} 
positive bequests. Then $\widehat{\tau}^{D}=0$.

Proof. Because $\frac{\partial \gamma^{D}(\beta . \tau, \xi)}{\partial \tau}=\beta \frac{\partial r(\tau, \xi)}{\partial \tau}<0$ we have that $\widehat{\tau}^{D}=0$.

In the infinitely-lived generations' environment, the growth maximizing policy is independent of the magnitude of the natural resource abundance; letting resource revenues work as input into production leads to higher growth rates, since the rate of return is highest, when direct transfers $\tau$ are zero.

Combining lemma 2 and 3 , it can be shown that when $\xi \leq \frac{\alpha^{2}}{1-\alpha}$, the growth maximizing policy is identical for the two growth regimes, namely zero direct transfers.

Proposition 5. Under growth maximizing policies, a resource curse does not exist; increased natural resource abundance enhances growth.

Proof. See Appendix.

The growth maximizing spending policy depends on the strength of altruism. For high values of the altruism factor, in order to enhance growth, all resource revenues are invested in public services, and for low values of altruism, the growth maximizing policy is to allocate a share of the natural resource revenues as direct transfers to the young.

Growth maximizing policies may, however, suffer from another potential problem: dynamic inefficiency. When bequests are absent, the only way for the young to provide for themselves when old, is to save, which they may do even if the interest rate is very low. In this case, transferring resources from the young generation to the old generation is Pareto efficient.

Dynamic inefficiency in endogenous growth models occurs when the competitive real rate of interest falls short of the growth rate (King and Ferguson 1993). This 
condition corresponds to $\beta^{*}(\cdot)>1 .{ }^{16}$ Under growth maximizing policies, if, e.g., $\widehat{\pi}^{O}=\widehat{\tau}^{O}=1-\alpha-\frac{\alpha^{2}}{\xi}$, then $\beta^{*}\left(\widehat{\pi}^{O}, \widehat{\tau}^{O}, \xi\right)=\frac{\rho(\alpha+\xi)}{1+\rho} ;$ therefore, in this case, growth maximizing policies trigger dynamic inefficiency when $\rho>\frac{1}{\alpha+\xi-1}$ and bequests are absent.

\subsection{Young and Old Policies}

In this section, either the young or the old decide a policy, which is implemented by the government. The policy remains unaltered in perpetuity, ${ }^{17}$ and the policy decision is made at the beginning of some arbitrary initial period. After determining the policy, the young earn a wage, decide their savings knowing whether they receive a direct transfer from the government or bequests or both. The old receive a return on their savings, possibly receive a direct transfer from the government, and possibly leave bequests.

At time $t$, the utility of a young person is given as in (9):

$$
\begin{aligned}
\bar{V}_{t} & =\sum_{i=0}^{\infty} \beta^{i}\left[\ln \left(c_{1 t+i}\right)+\rho \ln \left(c_{2 t+1+i}\right)\right] \\
& =\frac{1}{1-\beta}\left\{\ln \left(c_{1 t}\right)+\rho \ln \left(c_{2 t}\right)+\left(\frac{\beta+\rho}{1-\beta}\right) \ln [1+\gamma]\right\} .
\end{aligned}
$$

The young derive utility of own consumption both as young and as old as well of consumption of their heirs. The old, on the other hand, only derive utility of own consumption as old and of consumption of their heirs:

$$
\begin{aligned}
\underline{V}_{t} & =\ln \left(c_{2 t}\right)+\sum_{i=0}^{\infty} \beta^{1+i}\left[\ln \left(c_{1 t+i}\right)+\rho \ln \left(c_{2 t+1+i}\right)\right] \\
& =\ln \left(c_{2 t}\right)+\frac{\beta}{1-\beta}\left\{\ln \left(c_{1 t}\right)+\rho \ln \left(c_{2 t}\right)+\left(\frac{\beta+\rho}{1-\beta}\right) \ln [1+\gamma]\right\} .
\end{aligned}
$$

Nevertheless,

\footnotetext{
${ }^{16} \gamma^{O}(\pi, \tau, \xi)>r(\tau, \xi) \Leftrightarrow \beta^{*}(\xi, \cdot)(1-a) f(\tau, \xi)-1>(1-a) f(\tau, \xi)-1 \Longleftrightarrow \beta^{*}(\xi, \cdot)>1$.

${ }^{17} \mathrm{~A}$ similar assumption is made in Alesina and Rodrik (1994).
} 
Proposition 6. The resource curse can exist when individuals have "a very small" altruism factor and spending policies are decided by an old generation.

Proof. See Appendix.

When the economy is borderline "non-altruistic," a policy decided by the old can trigger a resource curse. This may not be surprising in that the old generation care overridingly about its own consumption. Yet since the old receive a higher return to savings the higher the rate of return, the old do not claim all resource revenues. A part, if not all, of the revenue is still allocated to public services. Therefore, a "non-altruistic" economy ruled by the old is not automatically cursed.

Unfortunately, finding closed form solutions to the welfare maximizing policy of either generation cannot seem to be done in this model. By imposing an additional assumption to the problem, however, we are able to obtain such results.

Assumption 1. $\pi=\tau$. (Only the young receive direct transfers from the government.)

As the assumption exogenously determines the intergenerational allocation of potential direct transfers from the government, we refer to policies under assumption 1 as "quasi-endogenous" spending policies. In the following, young policies and old policies, i.e., the policies that the young or the old implement in order to maximize their welfare, are accordingly "quasi-endogenous" spending policies.

Assumption 1 is not binding when bequests are positive, since any change in the distribution of direct transfers of natural resource revenues across generations is offset by an opposite change in bequests. Assumption 1 does, however, affect the threshold altruism factor positively, which pushes economies towards being in the 
overlapping generations' regime.

Under assumption 1 , let $\bar{\tau}$ and $\underline{\tau}$ denote the policy that maximizes $\bar{V}_{t}$ and $\underline{V}_{t}$ given the size of the public budget given by the resource revenues. When $\bar{\tau}$ and $\underline{\tau}$ are zero, utility will increase further if the government collects lump-sum payments to increase the size of the public service. In this case, expanded public services increase the wage rate and the return to capital, which leads to an overall increase in utility. We analyze this possibility in the next section, but for now, the maximum size of the public service is bounded from above by inflows of natural resource revenues, as under growth maximizing policies.

Let $\bar{\tau}^{O}$ be the spending policy that maximizes young welfare subject to the public budget restriction when the economy is constrained to be without bequests, and let $\bar{\tau}^{D}$ be the spending policy that maximizes young welfare, also subject to the public budget restriction, when the economy is constrained to be with positive bequests. Moreover, define $\bar{\chi}^{O} \equiv \frac{1+\rho}{\rho(1-\beta)+1+\rho} \frac{1-\alpha}{\alpha}$ and $\bar{\chi}^{D} \equiv(1-\beta) \bar{\chi}^{O}$. Then, under assumption 1,

Lemma 4. Young policy when the economy is constrained to be in either growth regime:

$\bar{\tau}^{O}=\left\{\begin{array}{ll}0 & \text { if } \xi \bar{\chi}^{O} \leq \alpha \\ \frac{\xi \bar{\chi}^{O}-\alpha}{\xi\left(1+\bar{\chi}^{O}\right)} & \text { if } \xi \bar{\chi}^{O}>\alpha\end{array}\right.$ and $\bar{\tau}^{D}=\left\{\begin{array}{ll}0 & \text { if } \xi \bar{\chi}^{D} \leq 1-\beta(1-\alpha) \\ \frac{\xi \bar{\chi}^{D}-[1-\beta(1-\alpha)]}{\xi\left(1+\bar{\chi}^{D}\right)} & \text { if } \xi \bar{\chi}^{D}>1-\beta(1-\alpha)\end{array}\right.$.

Proof. See Appendix.

Likewise, subject to the public budget restriction, let $\underline{\tau}^{O}$ be the spending policy that maximizes welfare of the old generation when the economy is constrained to be without bequests, and let $\underline{\tau}^{D}$ be the spending policy that maximizes old welfare when the economy is constrained to be with positive bequests. Moreover, define 
$\underline{\chi}^{O} \equiv \frac{1+\rho}{\frac{(1-\beta)^{2}}{\beta}+\rho(1-\beta)+1+\rho} \frac{1-\alpha}{\alpha}$ and $\underline{\chi}^{D} \equiv \frac{(1-\beta)\left(\frac{1+\beta \rho}{\beta}\right)}{\frac{(1-\beta)^{2}}{\beta}+\rho(1-\beta)+1+\rho} \frac{1-\alpha}{\alpha}$. Then, under assumption 1,

LEMma 5. Old policy when the economy is constrained to be in either growth regime:

$\underline{\tau}^{O}=\left\{\begin{array}{ll}0 & \text { if } \xi \underline{\chi}^{O} \leq \alpha \\ \frac{\xi \underline{\chi}^{O}-\alpha}{\xi\left(1+\underline{\chi}^{O}\right)} & \text { if } \xi \underline{\chi}^{O}>\alpha\end{array}\right.$ and $\underline{\tau}^{I}=\left\{\begin{array}{ll}0 & \text { if } \xi \underline{\chi}^{D} \leq 1-\beta(1-\alpha) \\ \frac{\xi \underline{\chi}^{D}-[1-\beta(1-\alpha)]}{\xi\left(1+\underline{\chi}^{D}\right)} & \text { if } \underline{\xi} \underline{\chi}^{D}>1-\beta(1-\alpha)\end{array}\right.$.

Proof. See Appendix.

We notice that the young and old spending policies, $\bar{\tau}$ and $\underline{\tau}$, are both functions of the intertemporal and intergenerational discount factors as well as resource abundance; $\bar{\tau} \equiv \bar{\tau}(\bar{\chi}, \xi)$ and $\underline{\tau} \equiv \underline{\tau}(\underline{\chi}, \xi)$.

In both growth regimes, the marginal loss of increasing direct transfers is a decline in public service input into production, which channels into lower factor payments. The trade-off faced by the individual depends on the weights given in her welfare function, which, in turn, depends on whether she is young or old, and the growth dynamics. For example, when bequests are absent, all things equal, the young are more likely to implement a spending policy that involves direct transfers; $\bar{\chi}^{O}>\underline{\chi}^{O}, \frac{\partial \bar{\tau}^{O}(\cdot)}{\partial \bar{\chi}^{O}}>0$ and $\frac{\partial \underline{\tau}^{O}(\cdot)}{\partial \underline{\chi}^{O}}>0$. The young generations value the utility of own consumption in their utility function undiscounted, whereas the old discount the offspring's utility of young consumption using the intergenerational discount factor. The marginal utility the young obtain from direct transfers is therefore higher and offsets higher marginal utility costs which the direct transfers impose.

Moreover, under assumption 1,

Proposition 7. Within either growth regime, a resource curse does not exist 
under either young or old policies; increased natural resource abundance enhances growth.

Proof. See Appendix.

Furthermore, it is straightforward to show that under assumption 1, increased natural resource abundance increases welfare of the young and the old under both a young and an old policy, when the growth regime remains unaltered. Under both policies, $\frac{\partial c_{1 t}^{O}(\xi, \cdot)}{\partial \xi}>0, \frac{\partial c_{1 t}^{D}(\xi, \cdot)}{\partial \xi}>0, \frac{\partial c_{2 t}^{O}(\xi, \cdot)}{\partial \xi}>0, \frac{\partial c_{2 t}^{D}(\xi, \cdot)}{\partial \xi}>0$, and by proposition 7 , $\frac{\partial \gamma^{O}(\xi, \cdot)}{\partial \xi}>0$ and $\frac{\partial \gamma^{D}(\xi, \cdot)}{\partial \xi}>0$, so by $(36)$ and $(38)$ we have that within either regime $\frac{\partial \underline{V}_{t}}{\partial \xi}>0$ and $\frac{\partial \bar{V}_{t}}{\partial \xi}>0 \forall \pi=\tau$ and $0 \leq \tau<1$.

Lemma 4 and 5 imply that young and old policies are likely to vary, which means that also growth rates may vary across political regimes. An economy under either a young or an policy may grow faster in either regime. We notice also that young and old policies differ from growth maximizing policies (derived in lemma 2 and 3), when direct transfers are present. On the other hand, policies decided by the young, the old, as well as growth maximizing policies are coincident, when direct transfers are zero.

\subsubsection{Growth Regime Shifts and the Resource Curse}

The "quasi-endogenous" spending policies of an economy ruled by the young or the old laid out in lemma 4 and 5 are policies which are constrained by presence or absence of bequests. This section illustrates that the young or the old may be able to, through their policies, determine whether bequests are present or not, and unlike growth maximizing policies, young or old policies can imply a resource curse.

It suffices to analyze either of the above "quasi-endogenous" policies, since the 
mechanism is the same. We choose to analyze an economy ruled by the young and apply a numerical example given by $\beta=0.3,{ }^{18} \alpha=0.33$ and $\rho=0.55,{ }^{19}$ so that $\bar{\chi}^{O}=1.60$ and $\bar{\chi}^{D}=1.12$ by lemma 4 .

For the parameter values chosen for this example, a young policy, which is constrained by positive bequests, involves positive transfers when resource abundance is high (when the condition in lemma $4, \xi \bar{\chi}^{D}>1-\beta(1-\alpha)$, is fulfilled) and, accordingly, $\beta^{*}\left(\bar{\tau}^{D}(\xi, \cdot), \xi\right)$ grows as parents must be increasingly altruistic to leave positive bequests. The possibility of positive bequests remains, however, since $\beta^{*}\left(\bar{\tau}^{D}(\xi, \cdot), \xi\right)<\beta$ for all values of $0<\xi<1$ in the example. Yet at a certain level of resource abundance, the economy could also be in the overlapping generations' growth regime. At these levels of resource abundance, direct transfers under a no bequest constrained young policy would involve transfers so high that parents would not leave bequests. Interestingly, therefore, the economy, for values of $\xi$ high enough, could be on a growth path with zero or with positive bequests. The young, therefore, must compare welfare levels to determine the regime in which to set young policy which is not constrained by presence or absence of bequests.

Fig. 1 maps utility levels of a representative young individual under either growth regime along with the corresponding growth rates at different values of $\xi$. Both utility levels and growth rates of either regime increase the higher $\xi$. We only map utility and growth rate in the overlapping generations' regime for "relevant" values of $\xi$, i.e., when the economy could be in this regime under young policy.

Utility levels are illustrated by the thick lines and thus the line, which crosses from below, illustrates utility levels for the overlapping generations regime, whereas

\footnotetext{
${ }^{18}$ This value is taken from Croix and Michel $(2002,255)$.

${ }^{19}$ Assuming each period is 30 years, this corresponds to an annual discount rate of 2 percent.
} 
the other - kinked - line illustrates utility levels for the dynastic regime. The kink occurs then direct transfers become positive. At this point, a smaller share of the resource revenue is allocated to productive public services and the direct transfers from the government to the young are offset by a decline in parents' bequests. Therefore, the growth rate increases at a slower rate when $\xi$ gets higher. The fact that both consumption as young and old, as well as the growth rate, enters the welfare function of the young given in (36), explains the kink.

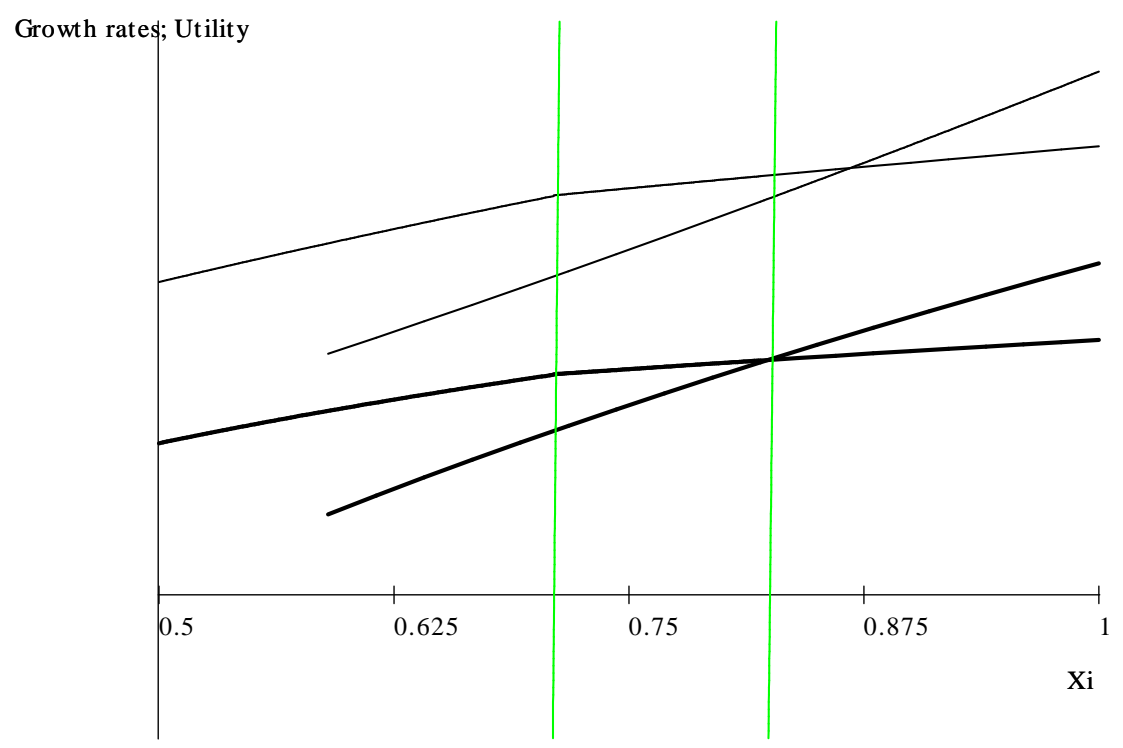

Figure 1. Growth rates and utility levels for a young person under different policies considered by a young policy maker at different levels of natural resource abundance.

Growth rates are illustrated by the thin lines in fig. 1. The line, which starts at $\xi=0.5$, illustrates the growth rate for the dynastic regime, and the line, which crosses it from below, illustrates the growth rate under the no bequest regime. Generally, the growth rates vary across growth regimes, and, we notice, that the dynastic growth rate has the kink that corresponds to the level of revenues at which 
positive direct transfers set in. Comparing utility levels, as $\xi$ becomes higher, there is a utility gain for the young person from shifting from the dynastic regime to overlapping generations regime. By shifting growth regime, as natural resources become more abundant, the young receives a larger direct transfer from the government, which, despite at the expense of bequests, leads to higher consumption levels as young, and, despite the drop in the growth rate, in higher welfare levels.

Indeed, the depicted economy experiences a decline in the growth rate, when, in response to higher resource revenues, the young policy maker decides a policy which shifts the economy from the dynastic regime to the overlapping generations' regime with a lower growth rate.

\section{The Optimal Policy}

This section introduces a social planner, who's objective is to maximize welfare, $W_{t}$, of current and all future generations. The role for a social planner is threefold as the model has three sources of inequality between the social planner equilibrium and the market equilibrium. First, the positive effect on GDP from man-made production, in the form of increased resource revenue, is external to the producers leading to under-saving and under-accumulation of capital. Second, in the market equilibrium, investments in the public service are restricted by the available budget, namely the resource revenues, and there may be economies for which an optimal size of public service flow is not feasible. Lastly, we noted that when bequests are absent, the competitive equilibrium may be dynamically inefficient.

Welfare at time $t=0, W_{0}$, is given as a weighted sum of current and future utilities of the members of society by a social welfare function that can be presented 
as

$$
W_{0}=\rho \ln \left(c_{20}\right)+\sum_{t=0}^{\infty} \beta^{t+1}\left[\ln \left(c_{1 t}\right)+\rho \ln \left(c_{2 t+1}\right)\right]
$$

where $\beta$ is the is the planner's intergenerational discount factor. ${ }^{20}$

The social planner runs a balanced budget, and the resource constraint is the same as in the market economy, given by

$$
(1+\xi) y_{t}=c_{1 t}+c_{2 t}+k_{t+1}+g_{t}
$$

with initial capital $k_{0}>0$. The variable $g_{t}$ denotes the level of public service chosen by the social planner. Using this constraint, and substituting $c_{1 t}$, (39) can be rewritten as

$$
W_{0}=\rho \ln \left(c_{20}\right)+\sum_{t=0}^{\infty} \beta^{t+1} \ln \left\{\left[(1+\xi) y_{t}-c_{2 t}-k_{t+1}-g_{t}\right]+\rho \ln \left(c_{2 t+1}\right)\right\}
$$

The necessary transversality condition is given by

$$
\lim _{t \rightarrow \infty} \beta^{t} q_{t} k_{t}=0
$$

where $q_{t}$ is the shadow price of the capital stock. Equation (42) ensures that the discounted value of wealth tends to zero and that (39) converges (Croix and Michel $2002,252)$.

The solution to the social planners problem is found by differentiating (41) with respect to $c_{2 t}, k_{t+1}$, and $g_{t}$. We obtain the following conditions satisfied for all periods, $t$ :

$$
\frac{\beta}{c_{1 t}}=\frac{\rho}{c_{2 t}}
$$

and

$$
\frac{1}{c_{1 t}}=\frac{\beta\left[(1+\xi) \frac{\partial y_{t}}{\partial k_{t}}\right]}{c_{1 t+1}} .
$$

\footnotetext{
${ }^{20}$ For simplicity, the private intergenerational discount factor equals that of the social planner. In principle, they could differ.
} 
Equation (43) says that the ratio between the utility of consumption of the young and the old generation must equal the ratio between the intertemporal and intergenerational discount factor. Unlike the solution to the altruistic generations' problem, this equation must hold, since otherwise welfare would increase by shifting consumption across generations. Equation (44) says that the ratio between utility of young consumption in two consecutive periods must equal the intergenerational discount rate multiplied with the gross return on capital, since this is the return to savings. As capital fully depreciates, the social return to capital, $r_{t}^{s p}$, is

$$
(1+\xi) \frac{\partial y_{t}}{\partial k_{t}}=(1+\xi)(1-\alpha) \frac{y_{t}}{k_{t}}=1+r_{t}^{s p},
$$

so (44) can be rewritten as

$$
\frac{1}{c_{1 t}}=\frac{\beta\left(1+r_{t}^{s p}\right)}{c_{1 t+1}}
$$

The social planner chooses an optimal public service flow that satisfies

$$
(1+\xi) \frac{\partial y_{t}}{\partial g_{t}}=1
$$

In optimum, the marginal benefit of increasing the public service, the rise in manmade output plus resource revenues, is exactly equal to the cost of doing so. By use of (4), (47) can be expressed as

$$
g_{t}=\alpha(1+\xi) y_{t}
$$

As $g_{t}$ is a constant fraction of $y_{t}$, in optimum, the public spending output ratio is constant. Applying this expression of $g_{t}$, we can rewrite (4) as

$$
y_{t}=A k_{t}\left[\frac{\alpha(1+\xi) y_{t}}{k_{t}}\right]^{\alpha} \Leftrightarrow y_{t}=\left[A \alpha^{\alpha}(1+\xi)^{\alpha}\right]^{\frac{1}{1-\alpha}} k_{t} \equiv f^{s p}(\xi) k_{t} .
$$

Using (49), we can express $r_{t}^{s p}$ from (45)

$$
r_{t}^{s p}=(1+\xi)(1-\alpha) f^{s p}(\xi)-1 \equiv r^{s p}(\xi)
$$


By (46), the growth rate in the consumption of the young generation, $\gamma_{t+1}$, is

$$
\gamma_{t+1}=\beta\left[1+r^{s p}(\xi)\right]-1 \equiv \gamma(\xi)
$$

We define an optimal balanced growth path as a path along which $c_{1 t}, c_{2 t}, k_{t}, y_{t}$, $g_{t}$, and $e_{t}$ grow at a constant relative rates, $\gamma(\xi)$, at all periods $t>0$. From (49) it follows that capital grows at the same rate as output. Since resource revenues are given as a fixed fraction of output (in (1)), it follows immediately that the inflow of natural resource revenues grows at the same rate as output. Moreover, as public services are given as a fixed fraction of total resource revenues (in (57)), also public services grow at the rate of output.

By the resource constraint (in (40)) and (50) it must be that if capital and output grow at the same rate, then this rate equals that of consumption. From (43), we know that the ratio of young and old consumption is constant; thus, old consumption grows at the same rate as young consumption.

It can be shown that $q_{t}$ equals $\frac{1}{c_{1 t-1}}$ (Croix and Michel 2002, 103) and therefore decreases at the rate $\gamma(\xi)$, and the transversality condition in (16) simplifies to $\beta<1$. To ensure non-negative growth, we assume that $\beta \geq \frac{1}{1+r^{s p}(\xi)}$. The economy has no transitional economics; $c_{1 t}, c_{2 t}, k_{t}, y_{t}, g_{t}$, and $e_{t}$ are an optimal solution to the social planners problem and grow at the same rate along a balanced growth path at all periods, where (51) characterizes the balanced growth path. Along such a path, equilibrium is given by

$$
\begin{aligned}
c_{1 t} & =\frac{\beta(1-\beta)}{\beta+\rho}(1+\xi)(1-\alpha) f^{s p}(\xi) k_{t} \equiv c_{1 t}(\xi), \\
c_{2 t} & =\frac{\rho(1-\beta)}{\beta+\rho}(1+\xi)(1-\alpha) f^{s p}(\xi) k_{t} \equiv c_{2 t}(\xi), \\
k_{t+1} & =\beta(1+\xi)(1-\alpha) f^{s p}(\xi) k_{t} \equiv k_{t+1}(\xi),
\end{aligned}
$$


with $k_{0}>0$ given.

\subsection{Decentralization}

We proceed to show how the social planner may decentralize the optimal solution just derived. Decentralization requires three policy instruments since one externality has to be internalized, public services have to be financed, and the competitive equilibrium may be dynamically inefficient.

To internalize the spillover effect from man-made production onto resource revenues, the social planner subsidizes the firms with the resource inflow. The representative firm solves

$$
\max _{K_{t}, L}\left\{(1+\xi) A K_{t}^{1-\alpha}\left(g_{t} L\right)^{\alpha}-r_{t} K_{t}-w_{t} L\right\}
$$

taking $g_{t}, r_{t}$, and $w_{t}$ as given. Hence, from the standpoint of the firm,

$$
r_{t}=(1+\xi)(1-\alpha) f^{s p}(\xi)-1=r^{s p}(\xi),
$$

and the private marginal return to capital coincides with the social marginal return.

As resource revenues are allocated to the firms, the social planner collects lumpsum taxes to invest in the public service. It is convenient to let the tax be a share of GDP, so

$$
\widetilde{\tau}(1+\xi) y_{t}=g_{t},
$$

where the lump-sum tax rate, $\widetilde{\tau}$, is a constant. It follows from the balanced budget that $0<\widetilde{\tau}<1$. The social planner collects a share, $\tilde{\pi}$, of the tax payments from the young generation and the rest, $(1-\tilde{\pi})$, from the old generation. An altruistic individual is now faced with the following budget constraint:

$$
c_{1 t}+s_{t}=b_{t}+w_{t}-\tilde{\pi} g_{t},
$$


when young, and

$$
c_{2 t+1}+b_{t+1}=\left[1+r^{s p}(\xi, \cdot)\right] s_{t}-(1-\tilde{\pi}) g_{t+1},
$$

when old, where the real wage rate, $w_{t}$, can be derived from (55), and $b_{t} \geq 0$.

Lemma 6. Let $\tilde{\pi}$ and $\widetilde{\tau}$ be a policy that decentralizes the resource allocation chosen by the social planner. Then $\widetilde{\tau}=\alpha$ and $\widetilde{\pi}=1-\frac{1-\alpha}{\alpha} \frac{\beta(1+\rho)}{\beta+\rho}$.

Proof. The size of the public service is optimal when (48) equals (57). Hence, $\widetilde{\tau}=\alpha$.

The transfer which ensures the competitive consumption path equals the optimal consumption path when bequests are absent satisfies for old consumption, by (59) and (53), that

$$
\left[1+r^{s p}(\xi)\right] k_{t}-(1-\tilde{\pi}) g_{t}=\frac{\rho(1-\beta)}{\beta+\rho}(1+\xi)(1-\alpha) f^{s p}(\xi) k_{t}
$$

Substituting $r^{s p}(\xi), k_{t}$, and $g_{t}$, and solving for $\tilde{\pi}$, we find $\tilde{\pi}=1-\frac{1-\alpha}{\alpha} \frac{\beta(1+\rho)}{\beta+\rho}$.

When bequests are positive, they are given by

$$
b_{t}=(1+\xi) f^{s p}(\xi)\left[(1-\alpha) \frac{\beta(1+\rho)}{\beta+\rho}-\alpha(1-\tilde{\pi})\right] k_{t}
$$

which is positive for $\widetilde{\pi}>1-\frac{1-\alpha}{\alpha} \frac{\beta(1+\rho)}{\beta+\rho}$ and just zero when $\widetilde{\pi}=1-\frac{1-\alpha}{\alpha} \frac{\beta(1+\rho)}{\beta+\rho}$. As

$$
\frac{\partial b_{t}}{\partial \widetilde{\pi}}=\alpha(1+\xi) f^{s p}(\xi) k_{t}=g_{t}
$$

and as

$$
c_{2 t}=\left[1+r^{s p}(\xi)\right] k_{t}-(1-\tilde{\pi}) g_{t}-b_{t}
$$

so

$$
\frac{\partial c_{2 t}}{\partial \widetilde{\pi}}=0
$$


consumption is invariant to changes in $\widetilde{\pi}$ as long as $\widetilde{\pi} \geq 1-\frac{1-\alpha}{\alpha} \frac{\beta(1+\rho)}{\beta+\rho}$.

The share, $\tilde{\pi}$, thus provides a lower bound on how little the young may be taxed and still leave the parent at least indifferent between leaving bequests or not. When $1>\tilde{\pi}>0$, both the old and the young contribute to the public service. When $\tilde{\pi}=0$ only the old pay, and when $\tilde{\pi}<0$, only the old pay and the young generation receives a transfer. The share, $\tilde{\pi}$, cannot be higher than one, since then the young would have negative consumption.

As we expect, an optimal balanced growth path is dynamically efficient. When $\tilde{\pi}=1-\frac{1-\alpha}{\alpha} \frac{\beta(1+\rho)}{\beta+\rho}$, parents are indifferent about leaving bequests and $\beta=\beta^{*}$. Since $\beta<1$, the rate of return is higher than the rate of growth. Finally,

Proposition 8. Under optimal policies, welfare and growth increase when inflows of natural resource revenues increase.

Proof. By (39),

$$
W_{t}=\rho \ln c_{2 t}+\frac{1}{1-\beta}\left[\ln \left(c_{1 t}\right)+\rho \ln \left(c_{2 t+1}\right)+\frac{\beta(1+\rho)}{1-\beta} \ln (1+\gamma(\xi))\right]
$$

As $\frac{\partial \gamma(\xi)}{\partial \xi}>0$ we can conclude that the resource curse does not exist, and, further by $\frac{\partial c_{11}(\xi)}{\partial \xi}>0$ and $\frac{\partial c_{2 t}(\xi)}{\partial \xi}>0$, that $\frac{\partial W_{t}}{\partial \xi}>0$.

\section{Concluding Remarks}

Using an endogenous growth model with altruistic overlapping generations, we explain why nations may respond differently to natural resource abundance: Nations may be in different growth regimes that vary in how savings are affected by natural resource revenues. 
As first pointed out by Weil (1987), there is a threshold level of altruism which separates the two growth regimes. In our model, this threshold level of altruism, which is determined endogenously, is influenced by the allocation and the abundance of the resources. When parents' altruism is higher than the threshold altruism level, the bequest motive is operative, and resource abundance increases growth as well as welfare of either generation. Bequests interrupt the connection between direct transfers from the government and savings by allowing for offsetting intergenerational transfers from the old to the young. Therefore, savings are unaffected by how resource revenues are allocated across generations. In contrast, a resource curse may exist when the bequests motive is not strong enough that parents leave bequests. In this case, policies that allocate revenues to the old generation may harm savings of the young and, subsequently, growth. Yet the effect on the current generation's welfare is ambiguous; resource abundance may increase consumption levels which then (perhaps more than) compensates for reduced growth.

We also examine spending policies that are endogenously determined by a specific economic or political agenda. We find that a resource curse is avoided by growth maximizing policies. Under such policies, when bequests are absent, any direct transfers are given exclusively to the young generation. Higher resource abundance merely increases direct transfers, and, hence, savings and growth. When bequests are positive, all revenues are allocated to public services, since, in a dynastic regime, growth expands with more public services. Public services, in turn, expand with the revenue under this spending policy.

Instead, a resource curse may be triggered by gerontocracy when altruism is "very small." The old generation may prefer to allocate to itself direct transfers to 
an extent that higher resource abundance reduces savings of the young generation.

We also examine spending policies decided by a young and an old generation respectively. Unfortunately, however, we can only find closed form solutions to the welfare maximizing spending policy of either generation when the old generation is excluded from receiving direct transfers. In this case, however, a decline in growth rates caused by increased resource abundance implies an increase in welfare. Thus, the general use of the term "poor economic performance" in relation to slower economic growth rates may be misleading. Nevertheless, by solving the social planner's problem, we show that under optimal polices there can never be a resource curse as defined here.

Further theoretical work may seek to endogenize the policy decision which is modeled exogenously in this model. Such models will add to the literature on political economy explanations for the resource curse. Another extension is to examine other allocations of the resource revenue. In particular, a model in which the resource revenue is used only as direct transfers as suggested by Sala-i-Martin and Subramanian (2003) may lead to more explicit solutions. In addition, research into what factors, for instance, life-expectancy or fertility, that influence the strength of the bequest motive may be helpful in identifying how nations are affiliated with growth regimes, or development stages.

\section{Appendix}

\subsection{Proof of Proposition 2}

We prove proposition 2 by presenting parameter combinations for which $\frac{\partial \gamma^{O}(\cdot)}{\partial \xi}<0$ and, for both the young and the old generation, $\frac{\partial V_{t}}{\partial \xi}>0$. 
Utility of a representative young individual at time $t$ in the overlapping generations' regime, $\bar{V}_{t}^{O}$, is given as

$$
\bar{V}_{t}^{O}=\frac{1}{1-\beta}\left\{\ln \left(c_{1 t}^{O}\right)+\rho \ln \left(c_{2 t}^{O}\right)+\left[\frac{\beta+\rho}{1-\beta}\right] \ln \left(1+\gamma^{O}(\xi, \cdot)\right)\right\}
$$

(this equation is the same as (36) in the main text), where

$$
\frac{\partial \bar{V}_{t}^{O}}{\partial \xi}=\frac{1}{1-\beta}\left\{\frac{\frac{\partial c_{1 t}^{O}}{\partial \xi}}{c_{1 t}^{O}}+\frac{\rho \frac{\partial c_{2 t}^{O}}{\partial \xi}}{c_{2 t}^{O}}+\left[\frac{\beta+\rho}{1-\beta}\right] \frac{\frac{\partial \gamma^{O}(\xi, \cdot)}{\partial \xi}}{1+\gamma^{O}(\xi, \cdot)}\right\} .
$$

Utility of a representative old individual at time $t$ in the overlapping generations' regime, $\underline{V}_{t}^{O}$, is given as

$$
\underline{V}_{t}^{O}=\ln \left(c_{2 t}^{O}\right)+\frac{\beta}{1-\beta}\left\{\ln \left(c_{1 t}^{O}\right)+\rho \ln \left(c_{2 t}^{O}\right)+\left[\frac{\beta+\rho}{1-\beta}\right] \ln \left(1+\gamma^{O}(\xi, \cdot)\right)\right\}
$$

(this equation is the same as (38) in the main text), where

$$
\frac{\partial \underline{V}_{t}^{O}}{\partial \xi}=\frac{\frac{\partial c_{1 t}^{O}}{\partial \xi}}{c_{1 t}^{O}}+\frac{\beta}{1-\beta}\left\{\frac{\frac{\partial c_{1 t}^{O}}{\partial \xi}}{c_{1 t}^{O}}+\frac{\rho \frac{\partial c_{2 t}^{O}}{\partial \xi}}{c_{2 t}^{O}}+\left[\frac{\beta+\rho}{1-\beta}\right] \frac{\frac{\partial \gamma^{O}(\xi, \cdot)}{\partial \xi}}{1+\gamma^{O}(\xi, \cdot)}\right\} .
$$

When $\pi=0$, then

$$
\frac{\frac{\partial c_{1 t}^{O}}{\partial \xi}}{c_{1 t}^{O}}=\frac{\alpha}{(1-\alpha) \xi}+\frac{\tau}{1-\alpha+\tau \xi}-\frac{\tau}{(1+\rho)(1-\alpha)+\tau \xi},
$$

and

$$
\frac{\frac{\partial c_{2 t}^{O}}{\partial \xi}}{c_{2 t}^{0}}=\frac{\alpha}{(1-\alpha) \xi}+\frac{\tau}{1-\alpha+\tau \xi}
$$

and

$$
\frac{\frac{\partial \gamma^{O}(\xi, \cdot)}{\partial \xi}}{1+\gamma^{O}(\xi, \cdot)}=\frac{\alpha}{(1-\alpha) \xi}-\frac{\tau}{(1+\rho)(1-\alpha)+\tau \xi} .
$$

Substituting (62), (63), and (64) in (60) and (61) gives

$$
\frac{\partial \bar{V}_{t}^{O}}{\partial \xi}=\frac{1}{1-\beta}\left\{\frac{\alpha\left[1+\rho+\frac{\beta+\rho}{1-\beta}\right]}{(1-\alpha) \xi}+\frac{(1+\rho) \tau}{1-\alpha+\tau \xi}-\frac{\tau\left[\frac{1+\rho}{1-\beta}\right]}{(1+\rho)(1-\alpha)+\tau \xi}\right\},
$$


and

$$
\frac{\partial \underline{V}_{t}^{O}}{\partial \xi}=\frac{\alpha}{(1-\alpha) \xi}+\frac{\tau}{1-\alpha+\tau \xi}+\beta \frac{\partial \bar{V}_{t}^{O}}{\partial \xi} .
$$

We proceed by use of a numerical example in which $\alpha=0.2, \rho=0.5$, and $\beta=0.1$.

By the proof of lemma $1, \frac{\partial \gamma^{O}(\cdot)}{\partial \xi}<0 \Leftrightarrow \tau(1-2 \alpha)>\frac{(1-\alpha) \alpha}{\xi}(1+\rho)$. Hence, $\tau(1-2 \alpha)>\frac{(1-\alpha) \alpha}{\xi}(1+\rho)$ implies for the numerical example that

$$
\xi \tau>0.4
$$

Moreover, $\frac{\partial \bar{V}_{t}^{O}}{\partial \xi}>0$ implies

$$
\frac{6.5}{3}+\frac{6 \tau \xi}{0.8+\tau \xi}-\left(\frac{1}{0.9}\right) \frac{6 \tau \xi}{1.2+\tau \xi}>0
$$

and $\frac{\partial \underline{V}_{t}^{O}}{\partial \xi}>0$ implies

$$
\frac{1}{4 \xi}+\frac{\tau}{0.8+\tau \xi}+0.1\left[\frac{6.5}{3}+\frac{6 \tau \xi}{0.8+\tau \xi}-\left(\frac{1}{0.9}\right) \frac{6 \tau \xi}{1.2+\tau \xi}\right]>0
$$

Since $\frac{1}{4 \xi}+\frac{\tau}{0.8+\tau \xi}>0$ then $\frac{\partial \bar{V}_{t}^{O}}{\partial \xi}>0$ implies $\frac{\partial V_{t}^{O}}{\partial \xi}>0$. Hence, we can focus on welfare of the young generation. We complete the proof by providing an example where $\xi \tau>0.4$ and $\frac{6.5}{3}+\frac{6 \tau \xi}{0.8+\tau \xi}-\left[\frac{1}{0.9}\right] \frac{6 \tau \xi}{1.2+\tau \xi}>0$. For example, $\xi \tau=0.5>0.4$ satisfies the first condition, and $\xi \tau=0.5 \Rightarrow \frac{6.5}{3}+\frac{3}{1.2}-\left(\frac{1}{0.9}\right) \frac{3}{1.7}=2.7058>0$ satisfies the second condition. Hence, $\xi \tau=0.5$ satisfies $\frac{\partial \gamma^{O}(\cdot)}{\partial \xi}<0, \frac{\partial \bar{V}_{t}^{O}}{\partial \xi}>0$ and $\frac{\partial V_{t}^{O}}{\partial \xi}>0$.

\subsection{Proof of Proposition 4}

Combining (34) and (25) gives $\gamma^{O}(\xi, \cdot)=\beta^{*}(\xi, \cdot)(1-a) f(\tau, \xi)-1$. Then,

$$
\frac{\partial \gamma^{O}(\xi, \cdot)}{\partial \xi}=\frac{\partial \beta^{*}(\xi, \cdot)}{\partial \xi}[(1-a) f(\tau, \xi, \cdot)]+\beta^{*}(\xi, \cdot)(1-a) \frac{\partial f(\tau, \xi)}{\partial \xi}
$$

By (29), we derive

$$
\frac{\partial \gamma^{D}(\xi, \cdot)}{\partial \xi}=\beta(1-a) \frac{\partial f(\tau, \xi)}{\partial \xi}
$$


There are three situations: $\frac{\partial \beta^{*}(\xi, \cdot)}{\partial \xi}=0, \frac{\partial \beta^{*}(\xi, \cdot)}{\partial \xi}>0$, and $\frac{\partial \beta^{*}(\xi, \cdot)}{\partial \xi}<0$.

First, when $\frac{\partial \beta^{*}(\xi, \cdot)}{\partial \xi}=0$, the growth regime remains unaltered by higher resource revenues and $\frac{\partial \gamma^{O}(\xi, \cdot)}{\partial \xi}>0$ and $\frac{\partial \gamma^{D}(\xi, \cdot)}{\partial \xi}>0$ since $\frac{\partial f(\tau, \xi)}{\partial \xi}>0$.

Second, when $\frac{\partial \beta^{*}(\xi,)}{\partial \xi}>0$, if an economy changes regime, it changes from a dynastic to an overlapping generations regime. When $\beta=\beta^{*}(\xi, \cdot)$, by definition, $\gamma^{O}(\xi, \cdot)=\gamma^{D}(\xi, \cdot)$. At this point, $\frac{\partial \gamma^{O}(\xi, \cdot)}{\partial \xi}>\frac{\partial \gamma^{D}(\xi, \cdot)}{\partial \xi}$, and $\gamma^{O}(\xi, \cdot) \operatorname{crosses} \gamma^{D}(\xi, \cdot)$ from below. Hence, when the economy shifts from $\gamma^{D}(\xi, \cdot)$ to $\gamma^{O}(\xi, \cdot)$, the economy grows faster on the new growth path than on the old growth path.

Third, when $\frac{\partial \beta^{*}(\xi, \cdot)}{\partial \xi}<0$, if the economy changes regime, it changes from an overlapping generations regime to a dynastic regime. Again, when $\beta=\beta^{*}(\xi, \cdot)$, by definition, $\gamma^{O}(\xi, \cdot)=\gamma^{D}(\xi, \cdot)$. In this point, $\frac{\partial \gamma^{O}(\xi, \cdot)}{\partial \xi}<\frac{\partial \gamma^{D}(\xi, \cdot)}{\partial \xi}$, and $\gamma^{O}(\xi, \cdot)$ crosses $\gamma^{D}(\xi, \cdot)$ from above. Hence, when the economy shifts from $\gamma^{O}(\xi, \cdot)$ to $\gamma^{D}(\xi, \cdot)$, the economy grows faster on the new growth path than on the old growth path.

\subsection{Proof of Lemma 2}

We consider the problem

$$
\max _{\pi, \tau} \gamma^{O}(\pi, \tau, \xi) \text { subject to } \pi \geq 0, \pi \leq \tau \text {, and } \tau<1 \text {. }
$$

The growth rate, $\gamma^{O}(\pi, \tau, \xi)$, is twice differentiable and concave and the restrictions are all linear. The Kuhn-Tucker conditions are thus both necessary and sufficient. The Lagrangian is

$$
\mathcal{L}=\frac{\rho(1-\alpha) f(\cdot)(\alpha+\pi \xi)}{(1+\rho)(1-\alpha)+(\tau-\pi) \xi}-1+\lambda_{1} \pi-\lambda_{2}(\pi-\tau)-\lambda_{3}(\tau-1)
$$


The five Kuhn-Tucker conditions are

$$
\begin{array}{r}
\frac{\rho(1-\alpha) \xi f(\cdot)}{(1+\rho)(1-\alpha)+(\tau-\pi) \xi}\left[1+\frac{\alpha+\pi \xi}{(1+\rho)(1-\alpha)+(\tau-\pi) \xi}\right]+\lambda_{1}-\lambda_{2}=0(65) \\
\frac{\rho(1-\alpha)(\alpha+\pi \xi)}{(1+\rho)(1-\alpha)+(\tau-\pi) \xi}\left[\frac{\partial f(\cdot)}{\partial \tau}-\frac{f(\cdot) \xi}{(1+\rho)(1-\alpha)+(\tau-\pi) \xi}\right]+\lambda_{2}-\lambda_{3}=0(66) \\
\lambda_{1} \geq 0, \pi \geq 0, \lambda_{1} \pi=0(67) \\
\lambda_{2} \geq 0, \pi \leq \tau, \lambda_{2}(\pi-\tau)=0(68) \\
\lambda_{3} \geq 0, \tau<1, \quad \lambda_{3}(\tau-1)=0(69)
\end{array}
$$

If $\pi=0$ and $\tau=0$ then $\lambda_{3}=0$. Combination of (65) and (66) yields

$$
\lambda_{1}=\frac{-\rho\left[\xi f(\cdot)+\frac{\partial f(\cdot)}{\partial \tau} \alpha\right]}{1+\rho} .
$$

Since $\lambda_{1} \geq 0, \frac{\alpha^{2}}{\xi} \geq 1-\alpha$ must be satisfied for this to be a solution. In this case,

$$
\lambda_{2}=\frac{\rho \xi f(\cdot)}{1+\rho}+\frac{\rho f(\cdot) \alpha \xi}{(1+\rho)^{2}(1-\alpha)}+\lambda_{1}>0
$$

which is then a solution.

If $\pi=0$ and $\tau>0$, then $\lambda_{2}=\lambda_{3}=0$. Hence, from (65)

$$
\lambda_{1}=-\frac{\rho(1-\alpha) \xi f(\cdot)}{(1+\rho)(1-\alpha)+\tau \xi}\left[1+\frac{\alpha}{(1+\rho)(1-\alpha)+\tau \xi}\right]
$$

which contradicts $\lambda_{1} \geq 0$.

If $\pi>0$ and $\tau>0$, then $\lambda_{1}=\lambda_{3}=0$. Hence, from (65) for this to be a solution, $\lambda_{2}>0$, and by (68), this requires $\pi=\tau$. Using (65) in (66), we have

$$
\frac{\partial f(\cdot)}{\partial \tau}(\alpha+\tau \xi)+\xi f(\cdot)=0
$$

which is satisfied when $\tau=1-\alpha-\frac{\alpha^{2}}{\xi}$.

We conclude that there are two solutions of the Kuhn-Tucker conditions: $\left(\pi, \tau, \lambda_{1}, \lambda_{2}, \lambda_{3}\right)=\left(1-\alpha-\frac{\alpha^{2}}{\xi}, 1-\alpha-\frac{\alpha^{2}}{\xi}, 0, \frac{\rho \xi f(\cdot)}{1+\rho}+\frac{\rho f(\cdot)(\alpha+\pi \xi) \xi}{(1+\rho)^{2}(1-\alpha)}, 0\right)$ if $\frac{\alpha^{2}}{\xi}<1-\alpha$, and 
$\left(\pi, \tau, \lambda_{1}, \lambda_{2}, \lambda_{3}\right)=\left(0,0, \frac{-\rho\left[\xi f(\cdot)+\frac{\partial f(\cdot)}{\partial \tau} \alpha\right]}{1+\rho}, \frac{\rho \xi f(\cdot)}{1+\rho}+\frac{\rho f(\tau, \xi) \alpha \xi}{(1+\rho)^{2}(1-\alpha)}-\frac{\rho\left[\xi f(\cdot)+\frac{\partial f(\cdot)}{\partial \tau} \alpha\right]}{1+\rho}, 0\right)$ if

$\frac{\alpha^{2}}{\xi} \geq 1-\alpha$.

\subsection{Proof of Proposition 5}

First, consider the economy without bequests. Let $\widehat{\gamma}^{O}$ denote the growth rate under a growth maximizing spending policy when the economy is without bequests, then

$$
\widehat{\gamma}^{O}=\widehat{\beta}^{*}(1-\alpha) \widehat{f}^{O}-1 \equiv \widehat{\gamma}^{O}\left(\widehat{\pi}^{O}, \widehat{\tau}^{O}, \xi\right)
$$

where $\widehat{\beta}^{*} \equiv \beta^{*}\left(\widehat{\pi}^{O}, \widehat{\tau}^{O}, \xi\right)=\frac{\rho\left(\alpha+\widehat{\tau}^{O} \xi\right)}{(1+\rho)(1-\alpha)}$ and $\widehat{f}^{O} \equiv f\left(\widehat{\tau}^{O}, \xi\right)$. Direct transfers may be zero or positive. When $\widehat{\pi}^{O}=\widehat{\tau}^{O}=0$, then

$$
\frac{\partial \widehat{\gamma}^{O}(\cdot)}{\partial \xi}=(1-\alpha)\left[\frac{\rho \alpha}{1+\rho} \frac{\partial \widehat{f}^{O}}{\partial \xi}\right]>0
$$

When, $\widehat{\pi}^{O}=\widehat{\tau}^{O}=1-\alpha-\frac{\alpha^{2}}{\xi}$, then

$$
\frac{d \widehat{\gamma}^{O}(\cdot)}{d \xi}=(1-\alpha)\left[\frac{\partial \widehat{\beta}^{*}}{\partial \widehat{\tau}^{O}} \frac{d \widehat{\tau}^{O}}{d \xi} \widehat{f}^{O}+\widehat{\beta}^{*} \frac{\partial \widehat{f}^{O}}{\partial \widehat{\tau}^{O}} \frac{d \widehat{\tau}^{O}}{d \xi}\right] .
$$

Since $\frac{\partial \widehat{\beta}^{*}}{\partial \widehat{\tau}^{O}} \frac{d \widehat{\tau}^{O}}{d \xi}>0$ and $\frac{\partial \widehat{f}^{O}}{\partial \widehat{\tau}^{O}} \frac{d \widehat{\tau}^{O}}{d \xi}>0$, we have that $\frac{d \widehat{\gamma}^{O}(\cdot)}{d \xi}>0$.

Second, let $\widehat{\gamma}^{D}$ denote the growth rate when the economy is dynastic, thus

$$
\widehat{\gamma}^{D}=\beta(1-\alpha) \widehat{f}^{D}-1 \equiv \widehat{\gamma}^{D}\left(\widehat{\tau}^{D}, \xi\right)
$$

where $\widehat{f}^{D} \equiv f\left(\widehat{\tau}^{D}, \xi\right)$. Since $\widehat{\tau}^{D}=0$,

$$
\frac{\partial \widehat{\gamma}^{D}(\cdot)}{\partial \xi}=\beta(1-\alpha) \frac{\partial \widehat{f}^{D}}{\partial \xi}>0
$$

Third, it follows immediately than when the economy is in a situation where policies decide whether bequests are absent or present, i.e.,

$$
\frac{\rho\left[\alpha+\left(1-\alpha-\frac{\alpha^{2}}{\xi}\right) \xi\right]}{(1+\rho)(1-\alpha)}>\beta<\frac{\rho \alpha}{(1+\rho)(1-\alpha)},
$$


policies will be such that the economy is in the regime which grows the fastest. Thus, there is no resource curse under growth maximizing policies, since both regimes grow faster at higher levels of $\xi$.

\subsection{Proof of Proposition 6}

We consider the problem

$$
\max _{\pi, \tau} \underline{V}_{t}^{O} \text { subject to } \pi \geq 0, \pi \leq \tau, \text { and } \tau<1 \text {. }
$$

First, we choose $\beta=0$, then $\underline{V}_{t}^{O}=\ln \left(c_{2 t}^{O}\right)=\ln \left\{f(\tau, \xi)[1-\alpha+(\tau-\pi) \xi] k_{t}^{O}\right\}$. Utility, $\underline{V}_{t}^{O}$, is twice differentiable and concave and the restrictions are all linear. The Kuhn-Tucker conditions are thus both necessary and sufficient. The Lagrangian is

$$
\mathcal{L}=\ln \left\{f(\tau, \xi)[1-\alpha+(\tau-\pi) \xi] k_{t}^{O}\right\}+\lambda_{1} \pi-\lambda_{2}(\pi-\tau)-\lambda_{3}(\tau-1)
$$

The five Kuhn-Tucker conditions are

$$
\begin{array}{r}
\frac{-\xi}{1-\alpha+(\tau-\pi) \xi}+\lambda_{1}-\lambda_{2}=0 \\
\frac{\xi \quad \frac{\alpha}{1-\alpha+(\tau-\pi) \xi}-\frac{1}{1-\alpha}+\tau}{1-\tau}-\lambda_{2}=0 \\
\lambda_{1} \geq 0, \pi \geq 0, \lambda_{1} \pi=0 \\
\lambda_{2} \geq 0, \pi \leq \tau, \lambda_{2}(\pi-\tau)=0 \\
\lambda_{3} \geq 0, \tau<1, \lambda_{3}(\tau-1)=0
\end{array}
$$

If $\pi=0$ and $\tau=0$, then $\lambda_{3}=0$. Combination of (71) and (72) then yields

$$
\lambda_{1}=\frac{\alpha}{1-\alpha}
$$

In this case,

$$
\lambda_{2}=\frac{-\xi}{1-\alpha}+\lambda_{1}
$$


Since $\lambda_{2} \geq 0$, when $\alpha \geq \xi$, this is then a solution.

If $\pi=0$ and $\tau>0$, then $\lambda_{2}=\lambda_{3}=0$. Hence, from (71),

$$
\lambda_{1}=\frac{\xi}{1-\alpha+\tau \xi}
$$

which is satisfied for $\tau>0$. From (72),

$$
\frac{\xi}{1-\alpha+\tau \xi}=\frac{\alpha}{1-\alpha} \frac{1}{1-\tau}
$$

which is satisfied for $\tau=(1-\alpha)\left(1-\frac{\alpha}{\xi}\right)$. As $\tau>0$, for this to be a solution $\alpha<\xi$.

If $\pi>0$ and $\tau>0$, then $\lambda_{1}=\lambda_{3}=0$. Since,

$$
\lambda_{2}=\frac{-\xi}{1-\alpha+(\tau-\pi) \xi},
$$

for this to be a solution, $\lambda_{2}>0$. This requires $\pi>\tau$, which contradicts $\pi \leq \tau$.

We conclude that there are two solutions of the Kuhn-Tucker conditions:

$\left(\pi, \tau, \lambda_{1}, \lambda_{2}, \lambda_{3}\right)=\left(0,0, \frac{\alpha}{1-\alpha}, \frac{\alpha-\xi}{1-\alpha}, 0\right)$ if $\alpha \geq \xi$, and $\left(0,(1-\alpha)\left(1-\frac{\alpha}{\xi}\right), \frac{\xi}{1-\alpha+\tau \xi}, 0,0\right)$ if $\alpha<\xi$. When $\pi=0$ and $\tau=(1-\alpha)\left(1-\frac{\alpha}{\xi}\right), \frac{\partial \gamma^{O}}{\partial \xi}=\frac{\rho f(\cdot)}{1+\rho+\xi-\alpha}\left(\frac{\alpha}{1-\alpha}+\frac{\xi}{\alpha+\xi}-\frac{\rho \xi}{1+\rho+\xi-\alpha}\right)$. Hence, when $\frac{\alpha}{1-\alpha}+\frac{\xi}{\alpha+\xi}<\frac{\rho \xi}{1+\rho+\xi-\alpha}, \frac{\partial \gamma^{O}}{\partial \xi}<0$.

Now, by continuity, this also holds for "sufficiently small" positive $\beta$ 's.

\subsection{Proof of Lemma 4}

We consider the problem

$$
\max _{\tau} \bar{V}_{t}\left(c_{1 t}, c_{2 t}, \gamma\right) \text { subject to } \tau \geq 0 \text { and } \tau<1
$$

given $\pi=\tau$, and where $\bar{V}_{t}\left(c_{1 t}, c_{2 t}, \gamma\right)$ is given by (36) in the main text with insertion of (26), (27) and (25) when the economy is constrained to be without bequests, and with insertion of (31), (32), and (29), when the economy is constrained to be with bequests. $\bar{V}_{t}$ is twice differentiable and concave and the restrictions are all linear. 
The Kuhn-Tucker conditions are thus both necessary and sufficient. The Lagrangian is

$$
\mathcal{L}=\bar{V}_{t}+\lambda_{1} \tau-\lambda_{2}(\tau-1)
$$

The three Kuhn-Tucker conditions are

$$
\begin{array}{r}
\frac{\partial \bar{V}_{t}}{\partial \tau}+\lambda_{1}-\lambda_{2}=0 \\
\lambda_{1} \geq 0, \tau \geq 0, \lambda_{1} \tau=0 \\
\lambda_{2} \geq 0, \tau<1, \lambda_{2}(\tau-1)=0
\end{array}
$$

where

$$
\frac{\partial \bar{V}_{t}}{\partial \tau}=\frac{1}{1-\beta}\left[\frac{1}{c_{1 t}} \frac{\partial c_{1 t}}{\partial \tau}+\frac{\rho}{c_{2 t}} \frac{\partial c_{2 t}}{\partial \tau}+\left(\frac{\beta+\rho}{1-\beta}\right) \frac{1}{1+\gamma(\tau, \cdot)} \frac{\partial \gamma(\tau, \cdot)}{\partial \tau}\right]
$$

Under assumption $1, \pi=\tau$, on a balanced growth path without bequests,

$$
\frac{1}{c_{1 t}^{O}} \frac{\partial c_{1 t}^{O}}{\partial \tau}=\frac{\xi}{\alpha+\tau \xi}-\frac{\alpha}{(1-\alpha)(1-\tau)}
$$

and

$$
\frac{1}{c_{2 t}^{O}} \frac{\partial c_{2 t}^{O}}{\partial \tau}=-\frac{\alpha}{(1-\alpha)(1-\tau)}
$$

and

$$
\frac{1}{1+\gamma^{O}(\tau, \cdot)} \frac{\partial \gamma^{O}(\tau, \cdot)}{\partial \tau}=\frac{\xi}{\alpha+\tau \xi}-\frac{\alpha}{(1-\alpha)(1-\tau)}
$$

Substituting (80), (81), and (82) into (79), we get

$$
\frac{\partial \bar{V}_{t}^{O}}{\partial \tau}=\frac{1}{1-\beta}\left[\left(\frac{1+\rho}{1-\beta}\right) \frac{\xi}{\alpha+\tau \xi}-\left(\rho+\frac{1+\rho}{1-\beta}\right) \frac{\alpha}{(1-\alpha)(1-\tau)}\right]
$$

By the Kuhn-Tucker conditions, if $\tau=0$, then $\lambda_{2}=0$, thus by (76),

$$
\lambda_{1}=-\frac{\partial \bar{V}_{t}^{O}}{\partial \tau} .
$$


Since $\lambda_{1} \geq 0,\left(\rho+\frac{1+\rho}{1-\beta}\right) \frac{\alpha}{1-\alpha} \geq\left(\frac{1+\rho}{1-\beta}\right) \frac{\xi}{\alpha}$ for this to be satisfied. Hence, $\alpha \geq \bar{\chi}^{O} \xi$ where $\bar{\chi}^{O} \equiv \frac{1+\rho}{\rho(1-\beta)+1+\rho} \frac{1-\alpha}{\alpha}(>0)$ for $\tau=0$ to be a solution.

If $\tau>0$, then $\lambda_{1}=\lambda_{2}=0$, thus by (76),

$$
\lambda_{1}=-\frac{\partial \bar{V}_{t}^{O}}{\partial \tau}=0
$$

Hence, $\left(\rho+\frac{1+\rho}{1-\beta}\right) \frac{\alpha}{(1-\alpha)(1-\tau)}=\left(\frac{1+\rho}{1-\beta}\right) \frac{\xi}{\alpha+\tau \xi}$, which implies $\tau=\frac{\xi \bar{\chi}^{O}-\alpha}{\xi\left(1+\bar{\chi}^{O}\right)}$, which is then a solution when $\xi \bar{\chi}^{O}>\alpha$.

We conclude that there are two solutions of the Kuhn-Tucker conditions: $\left(\bar{\tau}^{O}, \lambda_{1}, \lambda_{2}\right)=\left(0,-\frac{\partial \bar{V}_{t}^{O}}{\partial \tau}, 0\right)$ if $\alpha \geq \bar{\chi}^{O} \xi$ and $\left(\frac{\xi \bar{\chi}^{O}-\alpha}{\xi\left(1+\bar{\chi}^{O L G}\right)}, 0,0\right)$ if $\xi \bar{\chi}^{O}>\alpha$.

On a balanced growth path with positive bequests,

$$
\frac{1}{c_{1 t}^{D}} \frac{\partial c_{1 t}^{D}}{\partial \tau}=\frac{\xi}{1+\tau \xi-\beta(1-\alpha)}-\frac{\alpha}{(1-\alpha)(1-\tau)}
$$

and

$$
\frac{1}{c_{2 t}^{D}} \frac{\partial c_{2 t}^{D}}{\partial \tau}=\frac{\xi}{1+\tau \xi-\beta(1-\alpha)}-\frac{\alpha}{(1-\alpha)(1-\tau)}
$$

and

$$
\frac{1}{1+\gamma^{D}(\tau, \cdot)} \frac{\partial \gamma^{D}(\tau, \cdot)}{\partial \tau}=-\frac{\alpha}{(1-\alpha)(1-\tau)}
$$

Substituting (83), (84), and (85) into (79), we find

$$
\frac{\partial \bar{V}_{t}^{D}}{\partial \tau}=\frac{1}{1-\beta}\left\{\left[\frac{\xi}{1+\tau \xi-\beta(1-\alpha)}\right](1+\rho)-\left(\rho+\frac{1+\rho}{1-\beta}\right) \frac{\alpha}{(1-\alpha)(1-\tau)}\right\} .
$$

By the Kuhn-Tucker conditions, if $\tau=0$ then $\lambda_{2}=0$. By (76), then

$$
\lambda_{1}=-\frac{\partial \bar{V}_{t}^{D}}{\partial \tau} .
$$

Since $\lambda_{1} \geq 0,\left(\rho+\frac{1+\rho}{1-\beta}\right) \frac{\alpha}{(1-\alpha)} \geq\left[\frac{\xi}{1-\beta(1-\alpha)}\right](1+\rho)$ for this to be a solution. Hence, $1-\beta(1-\alpha) \geq \bar{\chi}^{D} \xi$ where $\bar{\chi}^{D} \equiv \frac{(1+\rho)(1-\beta)}{\rho(1-\beta)+1+\rho} \frac{1-\alpha}{\alpha}=(1-\beta) \bar{\chi}^{O}(>0)$ is a solution. 
If $\tau>0$, then $\lambda_{1}=\lambda_{2}=0$. By (76), then

$$
\lambda_{1}=-\frac{\partial \bar{V}_{t}^{D}}{\partial \tau}=0 .
$$

Hence, $\left[\frac{\xi}{1+\tau \xi-\beta(1-\alpha)}\right](1+\rho)=\left(\rho+\frac{1+\rho}{1-\beta}\right) \frac{\alpha}{(1-\alpha)(1-\tau)}$ which implies $\tau=\frac{\xi \bar{\chi}^{D}-[1-\beta(1-\alpha)]}{\xi\left(1+\bar{\chi}^{D}\right)}$.

Since $\tau>0$, for this to be a solution, $\xi \bar{\chi}^{D}>[1-\beta(1-\alpha)]$.

We conclude that there are two solutions of the Kuhn-Tucker conditions:

$\left(\bar{\tau}^{D}, \lambda_{1}, \lambda_{2}\right)=\left(0,-\frac{\partial \bar{V}_{t}^{D}}{\partial \tau}, 0\right)$ if $1-\beta(1-\alpha) \geq \bar{\chi}^{D} \xi$ and $\left(\frac{\xi \bar{\chi}^{D}-[1-\beta(1-\alpha)]}{\xi\left(1+\bar{\chi}^{D}\right)}, 0,0\right)$ if $\xi \bar{\chi}^{D}>[1-\beta(1-\alpha)]$.

\subsection{Proof of Lemma 5}

We consider the problem

$$
\max _{\tau} \underline{V}_{t}\left(c_{1 t}, c_{2 t}, \gamma\right) \text { subject to } \tau \geq 0 \text { and } \tau<1
$$

given $\pi=\tau$, and where $\underline{V}_{t}\left(c_{1 t}, c_{2 t}, \gamma\right)$ is given by (38) in the main text with insertion of (26), (27) and (25) when the economy is constrained to be without bequests, and with insertion of (31), (32), and (29), when the economy is constrained to be with bequests. $\underline{V}_{t}$, is twice differentiable and concave and the restrictions are all linear. The Kuhn-Tucker conditions are thus both necessary and sufficient. The Lagrangian is

$$
\mathcal{L}=\underline{V}_{t}+\lambda_{1} \tau-\lambda_{2}(\tau-1)
$$

The three Kuhn-Tucker conditions are

$$
\begin{array}{r}
\frac{\partial \underline{V}_{t}}{\partial \tau}+\lambda_{1}-\lambda_{2}=0 \\
\lambda_{1} \geq 0, \tau \geq 0, \quad \lambda_{1} \tau=0 \\
\lambda_{2} \geq 0, \tau<1, \lambda_{2}(\tau-1)=0
\end{array}
$$


where

$$
\frac{\partial \underline{V}_{t}}{\partial \tau}=\left(1+\frac{\beta \rho}{1-\beta}\right) \frac{1}{c_{2 t}} \frac{\partial c_{2 t}}{\partial \tau}+\frac{\beta}{1-\beta}\left[\frac{1}{c_{1 t}} \frac{\partial c_{1 t}}{\partial \tau}+\left(\frac{\beta+\rho}{1-\beta}\right) \frac{\frac{\partial \gamma(\tau, \cdot)}{\partial \tau}}{1+\gamma(\tau, \cdot)}\right]
$$

Substituting (80), (81), and (82) from the proof of Lemma 4 into (89), we find

$$
\frac{\partial \underline{V}_{t}^{O}}{\partial \tau}=\frac{\beta}{1-\beta}\left[\left(\frac{1+\beta \rho}{\beta}+\frac{\beta+\rho}{1-\beta}\right) \frac{-\alpha}{(1-\alpha)(1-\tau)}+\frac{1+\rho}{1-\beta} \frac{\xi}{\alpha+\tau \xi}\right]
$$

By the Kuhn-Tucker conditions, we have that if $\tau=0$, then $\lambda_{2}=0$. By (86), then

$$
\lambda_{1}=-\frac{\partial \underline{V}_{t}^{O}}{\partial \tau}
$$

Since $\lambda_{1} \geq 0,\left[1+\frac{\beta\left(\rho+\frac{1+\rho}{1-\beta}\right)}{1-\beta}\right] \frac{\alpha}{1-\alpha} \geq \frac{\beta}{1-\beta}\left(\frac{1+\rho}{1-\beta}\right) \frac{\xi}{\alpha}$ for this to be satisfied. Hence, $\alpha \geq \xi \underline{\chi}^{O}$, where $\underline{\chi}^{O} \equiv \frac{1+\rho}{\frac{(1-\beta)^{2}}{\beta}+\rho(1-\beta)+1+\rho} \frac{1-\alpha}{\alpha}(>0)$, for this to be a solution.

If $\tau>0$, then $\lambda_{1}=\lambda_{2}=0$. By (86) first condition then

$$
\lambda_{1}=-\frac{\partial \underline{V}_{t}^{O}}{\partial \tau}=0
$$

Thus, $\left[1+\frac{\beta\left(\rho+\frac{1+\rho}{1-\beta}\right)}{1-\beta}\right] \frac{\alpha}{(1-\alpha)(1-\tau)}=\frac{\beta}{1-\beta}\left(\frac{1+\rho}{1-\beta}\right) \frac{\xi}{\alpha+\tau \xi}$, which implies $\tau=\frac{\xi \underline{\chi}^{O}-\alpha}{\xi\left(1+\underline{\chi}^{O}\right)}$. Since $\tau>0$, for this to be a solution, $\xi \underline{\chi}^{O}>\alpha$.

We conclude that there are two solutions of the Kuhn-Tucker conditions: $\left(\underline{\tau}^{O}, \lambda_{1}, \lambda_{2}\right)=\left(0,-\frac{\partial \underline{V}_{t}^{O}}{\partial \tau}, 0\right)$ if $\alpha \geq \underline{\xi \chi}^{O}$ and $\left(\frac{\xi \underline{\chi}^{O}-\alpha}{\xi\left(1+\underline{\chi}^{O}\right)}, 0,0\right)$ if $\xi \underline{\chi}^{O}>\alpha$.

When bequests are positive, by use of (83), (84), and (85) from the proof of Lemma 4 , (89) is

$$
\frac{\partial \underline{V}_{t}^{D}}{\partial \tau}=\frac{1}{1-\beta}\left\{(1+\beta \rho) \frac{\xi}{1+\tau \xi-\beta(1-\alpha)}-\left[1+\beta \rho+\beta\left(\frac{\beta+\rho}{1-\beta}\right)\right] \frac{\alpha}{(1-\alpha)(1-\tau)}\right\}
$$

By the Kuhn Tucker conditions, if $\tau=0$, then $\lambda_{2}=0$, and then

$$
\lambda_{1}=-\frac{\partial \underline{V}_{t}^{D}}{\partial \tau}
$$


Since $\lambda_{1} \geq 0,\left[\left(\beta \rho+1+\beta\left(\frac{\beta+\rho}{1-\beta}\right)\right] \frac{\alpha}{1-\alpha} \geq(1+\beta \rho) \frac{\xi}{1-\beta(1-\alpha)}\right.$ for this to be satisfied.

Hence, $1-\beta(1-\alpha) \geq \xi \underline{\chi}^{D}$ where $\underline{\chi}^{D} \equiv \frac{1+\beta \rho}{\beta \rho+1+\beta\left(\frac{\beta+\rho}{1-\beta}\right)} \frac{1-\alpha}{\alpha}=\frac{\frac{1-\beta}{\beta}(1+\beta \rho)}{\frac{(1-\beta)^{2}}{\beta}+\rho(1-\beta)+1+\rho} \frac{1-\alpha}{\alpha}(>0)$ for this to be a solution.

If $\tau>0$, then $\lambda_{1}=\lambda_{2}=0$. By (86), then

$$
\lambda_{1}=-\frac{\partial \underline{V}_{t}^{D}}{\partial \tau}=0
$$

Thus, $\left[\left(\beta \rho+1+\beta\left(\frac{\beta+\rho}{1-\beta}\right)\right] \frac{\alpha}{(1-\alpha)(1-\tau)}=\frac{\xi(1+\beta \rho)}{1+\tau \xi-\beta(1-\alpha)}\right.$, which implies $\tau=\frac{\xi \underline{\chi}^{D}-[1-\beta(1-\alpha)]}{\xi\left(1+\underline{\chi}^{D}\right)}$. Since $\tau>0$, for this to be a solution, $\xi \underline{\chi}^{D}>[1-\beta(1-\alpha)]$.

We conclude that there are two solutions of the Kuhn-Tucker conditions: $\left(\bar{\tau}^{D}, \lambda_{1}, \lambda_{2}\right)=\left(0,-\frac{\partial \underline{V}_{t}^{D}}{\partial \tau}, 0\right)$ if $1-\beta(1-\alpha) \geq \xi \underline{\chi}^{D}$ and $\left(\frac{\xi \underline{\chi}^{D}-[1-\beta(1-\alpha)]}{\xi\left(1+\underline{\chi}^{D}\right)}, 0,0\right)$ if $\xi \underline{\chi}^{D}>[1-\beta(1-\alpha)]$.

\subsection{Proof of Proposition 7}

Bequests may be absent or present. Let $\bar{\gamma}^{O}$ denote the growth rate under a young spending policy when the economy is without bequests, then

$$
\bar{\gamma}^{O}=\bar{\beta}^{*}(1-\alpha) \bar{f}^{O}-1 \equiv \bar{\gamma}^{O}\left(\bar{\tau}^{O}, \xi\right)
$$

where $\bar{\beta}^{*} \equiv \beta^{*}\left(\bar{\tau}^{O}, \xi\right)=\frac{\rho\left(\alpha+\bar{\tau}^{O} \xi\right)}{(1+\rho)(1-\alpha)}$ and $\bar{f}^{O} \equiv f\left(\bar{\tau}^{O}, \xi\right)$, and let $\underline{\gamma}^{O}$ denote the growth rate under an old spending policy when the economy is without bequests, then

$$
\underline{\gamma}^{O}=\underline{\beta}^{*}(1-\alpha) \underline{f}^{O}-1 \equiv \underline{\gamma}^{O}\left(\underline{\tau}^{O}, \xi\right)
$$

where $\underline{\beta}^{*} \equiv \beta^{*}\left(\underline{\tau}^{O}, \xi\right)=\frac{\rho\left(\alpha+\underline{\tau}^{O} \xi\right)}{(1+\rho)(1-\alpha)}$ and $\underline{f}^{O} \equiv f\left(\underline{\tau}^{O}, \xi\right)$. Under both policies, direct transfers from the government may be zero or positive. We treat young and old policy in turn. Young policy (as given in lemma 4): When $\bar{\tau}^{O}=0$, then

$$
\frac{\partial \bar{\gamma}^{O}(\cdot)}{\partial \xi}=(1-\alpha)\left[\frac{\rho \alpha}{1+\rho} \frac{\partial \bar{f}^{O}}{\partial \xi}\right]>0
$$


when, $\bar{\tau}^{O}=\frac{\xi \bar{\chi}^{O}-\alpha}{\xi\left(1+\bar{\chi}^{O}\right)}$, then

$$
\frac{d \bar{\gamma}^{O}(\cdot)}{d \xi}=(1-\alpha)\left[\frac{\partial \bar{\beta}^{*}}{\partial \bar{\tau}^{O}} \frac{d \bar{\tau}^{O}}{d \xi} \bar{f}^{O}+\bar{\beta}^{*} \frac{\partial \bar{f}^{O}}{\partial \bar{\tau}^{O}} \frac{d \bar{\tau}^{O}}{d \xi}\right]
$$

Since $\frac{\partial \bar{\beta}^{*}}{\partial \bar{\tau}^{O}} \frac{d \bar{\tau}^{O}}{d \xi}>0$ and $\frac{\partial \bar{f}^{O}}{\partial \bar{\tau}^{O}} \frac{d \bar{\tau}^{O}}{d \xi}>0$, we have that $\frac{d \bar{\gamma}^{O}(\cdot)}{d \xi}>0$. Old policy (as given in lemma 5): When $\underline{\tau}^{O}=0$, then

$$
\frac{\partial \underline{\gamma}^{O}(\cdot)}{\partial \xi}=(1-\alpha)\left[\frac{\rho \alpha}{1+\rho} \frac{\partial \underline{f}}{\partial \xi}\right]>0
$$

when, $\underline{\tau}^{O}=\frac{\xi \underline{\chi}^{O}-\alpha}{\xi\left(1+\underline{\chi}^{O}\right)}$, then

$$
\frac{d \underline{\gamma}^{O}(\cdot)}{d \xi}=(1-\alpha)\left[\frac{\partial \underline{\beta}^{*}}{\partial \underline{\tau}^{O}} \frac{d \underline{\tau}^{O}}{d \xi} \bar{f}^{O}+\bar{\beta}^{*} \frac{\partial \underline{f}^{O}}{\partial \underline{\tau}^{O}} \frac{d \underline{\tau}^{O}}{d \xi}\right] .
$$

Since $\frac{\partial \underline{\underline{\beta}}^{*}}{\partial \underline{\tau}^{O}} \frac{d \underline{\tau^{O}}}{d \xi}>0$ and $\frac{\partial \underline{f}^{O}}{\partial \underline{\tau}^{O}} \frac{d \underline{\tau}^{O}}{d \xi}>0$, we have that $\frac{d \underline{\gamma}^{O}(\cdot)}{d \xi}>0$.

Let $\bar{\gamma}^{D}$ denote the growth rate under a young spending policy when the economy is dynastic, thus

$$
\bar{\gamma}^{D}=\beta(1-\alpha) \bar{f}^{D}-1 \equiv \bar{\gamma}^{D}\left(\bar{\tau}^{D}, \xi\right)
$$

where $\bar{f}^{D} \equiv f\left(\bar{\tau}^{D}, \xi\right)$. Likewise, let $\underline{\gamma}^{D}$ denote the growth rate under an old spending policy when the economy is dynastic, then

$$
\underline{\gamma}^{D}=\beta(1-\alpha) \underline{f}^{D}-1 \equiv \underline{\gamma}^{D}\left(\underline{\tau}^{D}, \xi\right)
$$

where $\underline{f}^{D} \equiv f\left(\underline{\tau}^{D}, \xi\right)$. Again, under both policies, direct transfers from the government may be zero or positive. We treat young and old policy in turn. Young policy (as given in lemma 4): When $\bar{\tau}^{D}=0$, then

$$
\frac{\partial \bar{\gamma}^{D}(\cdot)}{\partial \xi}=\beta(1-\alpha) \frac{\partial \bar{f}^{D}}{\partial \xi}>0,
$$

and when $\bar{\tau}^{D}=\frac{\xi \bar{\chi}^{D}-[1-\beta(1-\alpha)]}{\xi\left(1+\bar{\chi}^{D}\right)}$, then

$$
\frac{\partial \bar{\gamma}^{D}(\cdot)}{\partial \xi}=\beta(1-\alpha) \frac{\partial \bar{f}^{D}}{\partial \bar{\tau}^{D}} \frac{d \bar{\tau}^{D}}{d \xi}>0
$$


Old policy (as given in lemma 5): When $\underline{\tau}^{D}=0$ then

$$
\frac{\partial \underline{\gamma}^{D}(\cdot)}{\partial \xi}=\beta(1-\alpha) \frac{\partial f^{D}}{\partial \xi}>0,
$$

and when $\underline{\tau}^{D}=\frac{\xi \underline{\chi}^{D}-[1-\beta(1-\alpha)]}{\xi\left(1+\underline{\chi}^{D}\right)}$, then

$$
\frac{\partial \underline{\gamma}^{D}(\cdot)}{\partial \xi}=\beta(1-\alpha) \frac{\partial \underline{f}^{D}}{\partial \underline{\tau}^{D}} \frac{d \underline{\tau}^{D}}{d \xi}>0 .
$$

Thus, there is no resource curse under young or old policies when the growth regime remains unaltered.

\section{References}

Alesina, A., and D. Rodrik. 1994. Distributive politics and economic growth. Quarterly Journal of Economics 109(2): 465-490.

Auty, R. 1993. Sustaining development in mineral economies: The resource curse thesis. London: Routledge.

Auty, R., ed. 2001. Resource abundance and economic development. Oxford: Oxford University Press.

Barro, R. J. 1974. Are government bonds net wealth? Journal of Political Economy 82:1095-117.

Barro, R. J. 1990. Government spending in a simple model of endogenous growth. Journal of Political Economy 98 (2): 103-125.

Caballe, J. 1998. Growth effects of taxation under altruism and low elasticity of intertemporal substitution. The Economic Journal 108 (446): 92-104.

Cardia, E., and Ph. Michel. 2004. Altruism, intergenerational transfers of time and bequests. Journal of Economic Dynamics and Control 28:1681-1701. 
Chatterjee, S., G. Sakoulis, and S. J. Turnovsky. 2003. Unilateral capital transfers, public investment, and economic growth. European Economic Review 47:10771103.

Croix, D., and Ph. Michel. 2002. A theory of economic growth: Dynamics and policy in overlapping generations. Cambridge: Cambridge University Press.

Cordon, W. M., and J.P. Neary. 1982. Booming sector and de-industrialisation in small open economy. The Economic Journal 92:359-380.

Dasgupta, P. S., and G. M. Heal. 1979. Economic theory and exhaustible resources. Cambridge: Cambridge University Press.

Hannesson, R. 2001. Investing for sustainability: The management of mineral wealth. Norwell, Mass.: Kluwer Academic Publishers.

Hartwick, J. 1997. Intergenerational equity and the investing of revenues from exhaustible resources. American Economic Review 67:972-991.

King, I., and D. Ferguson. 1993. Dynamic inefficiency, endogenous growth and Ponzi games. Journal of Monetary Economics. 31:79-104.

Larsen, E. R. 2005. Are rich countries immune to the resource curse? Evidence from Norway's management of its oil riches. Resources Policy 30:75-86.

Lesink, R., and H. White. 2001. Are there negative returns to aid? Journal of Development Studies 37:42-65.

Matsen, E., and R. Torvik. 2005. Optimal dutch disease. Journal of Development Economics 78:494-515.

Mehlum, H., K. Moene, and R. Torvik (2006): Institutions and the resource curse. The Economic Journal 116:1-20.

Papyrakis, E., and R. Gerlagh. 2004. Natural resources, investment and long- 
term income. FEEM Working Paper, 87.04.

Robinson, J. A., and R. Torvik. 2005. White elephants. Journal of Public Economics 89:197-210.

Rodriguez, F., and J. D. Sachs. 1999. Why do resource abundant economies grow more slowly? A new explanation and application to Venezuela. Journal of Economic Growth 4:277-303.

Ross, M. 2004. What do we know about natural resources and civil war? Journal of Peace Research 41:337-356.

Ross, M. 2006. A closer look at oil, diamonds, and civil war. Annual Review of Political Science 9:265-300.

Sachs, J. D., and A. M. Warner. 1995. Natural resource abundance and economic growth. Development discussion paper, no. 517a, Harvard Institute for International Developments, Harvard University.

Sachs, J. D., and A. M. Warner. 1999. The big push, natural resource booms and growth. Journal of Development Economics 59:43-76.

Sachs, J. D., and A. M. Warner. 2001. Natural resources and economic development: The curse of natural resources. European Economic Review 45:827-838.

Sala-i-Martin, X., and A. Subramanian. 2003. Addressing the natural resource curse: An illustration from Nigeria. Working Paper 9804. NBER working paper series, National Bureau of Economic Research, Mass.

Sala-i-Martin, X., G. Doppelhofer, and R. I. Miller. 2004. Determinants of long-term growth: A Bayesian averaging of classical estimates (BACE) approach. American Economic Review 94 (4): 813-835.

Sandbu, M. E. 2006. Natural Wealth Accounts: A proposal for alleviating the 
natural resource curse. World Development 34 (7): 1153-1170.

Stevens, P. 2003. Resource impact: Curse or blessing? A literature survey. Journal of Energy Literature 9 (1): 3-42.

Stijns, J.-Ph. 2005. Natural resource abundance and economic growth revisited. Resources Policy 30:107-130.

Solow, R. 1974. Intergenerational equity and exhaustible resources. Review of Economic Studies 41:29-45.

Solow, R. 1986. On the intergenerational allocation of natural resources. Scandinavian Journal of Economics 88:143-154.

Tornell, A., and P. R. Lane. 1999. The voracity effect. American Economic Review 89:22-46.

Torvik, R. 2001. Learning by doing and the Dutch disease effect. European Economic Review 45:285-306.

Torvik, R. 2002. Natural resources, revenue seeking and welfare. Journal of Development Economics 67:455-470.

van Wijnbergen, S. 1984. The 'Dutch disease': A disease after all? The Economic Journal 94:41-55.

Weil, Ph. 1987. Love thy children: Reflections on the Barro debt neutrality theorem. Journal of Monetary Economics 19:377-391. 